Parallele Numerische Simulation für Physik und Kontinuumsmechanik

\author{
T. Eibner \\ J.M. Melenk
}

\title{
An adaptive strategy for $h p$-FEM based on testing for analyticity
}

Preprint SFB393/04-10

\begin{abstract}
We present an $h p$-adaptive strategy that is based on estimating the decay of the expansion coefficients when a function is expanded in $L^{2}$ orthogonal polynomails on a triangle or a tetrahedron. This method is justified by showing that the decay of the coefficients is exponential if and only if the function is analytic. Numerical examples illustrate the performance of this approach, and we compare it with two other $h p$-adaptive strategies.
\end{abstract}

Preprintreihe des Chemnitzer SFB 393 
Authors' addresses:

\section{Tino Eibner}

TU Chemnitz

Fakultät für Mathematik

D-09107 Chemnitz

Germany

email: t.eibner@mathematik.tu-chemnitz.de

Jens Markus Melenk

Department of Mathematics

PO Box 220

Reading RG6 6AX

United Kingdom

email: j.m.melenk@reading.ac.uk 


\section{Contents}

1 Introduction 2

2 Model problem and $h p$-FEM 3

3 Error indicator $\quad 7$

3.1 Performance of the error indicator . . . . . . . . . . . . . . 7

$4 \quad h p$-adaptive strategies $\quad 10$

4.1 Strategy I - Comparison of estimated and predicted error . . . . . . . . . . 11

4.2 Strategy II - Decay of Legendre expansion coefficients . . . . . . . . . . . . 12

4.3 Strategy III - Three fold algorithm . . . . . . . . . . . . . . . . . . . 13

4.4 Collection of numerical results . . . . . . . . . . . . . . . . . . . . . . . . 14

5 Analytic functions on tetrahedra $\quad 25$

5.1 Orthogonal polynomials on tetrahedra . . . . . . . . . . . . . . 26

5.2 Orthogonal polynomials and analytic functions . . . . . . . . . . . . . 26

5.3 Auxiliary Results . . . . . . . . . . . . . . . . . . . . . . . . 29 


\section{Introduction}

Over the last decades, adaptive solution strategies have become an accepted and more or less standard technique for solving partial differential equations via the finite element method. The main idea of all adaptive strategies is to start the computation with a lowdimensional approximation of the solution arising from a coarse grid on the computational domain in conjunction with low local approximation orders. Thereafter, in order to improve the accuracy of the approximation, an error indicator is employed to obtain information about the error distribution. Based on this error distribution, a suitable enlargement of the finite element space is chosen and a new approximation of higher accuracy is computed. The error of the new approximation is estimated and in case the approximation is not sufficiently accurate a new iteration of the adaptive loop is begun. In the adaptive $h$-FEM, the enlargement of the finite element space is simply done by subdividing into smaller elements all those elements that the error indicator has flagged as being tainted with a large error. However, in $h p$-FEM one has the option to split an element or to increase its approximation order. Thus, a main difficulty in $h p$-adaptivity is to decide whether to perform a $p$ - or an $h$-refinement for each element whose error is large. The importance of making the correct decisions is highlighted by the a priori analysis of I. Babuška and B. Guo (see [4] and the monograph [25] for a survey), where it is shown that for a large class of problems an exponential rate of convergence can be achieved if the meshes and polynomial degree distribution is chosen suitably.

Generally speaking, a local $p$-refinement is the more efficient method on elements where the solution is smooth. On the other hand, local $h$-refinement is the strategy suitable for regions where the solution is not smooth. Starting from this observation, most $h p$-adaptive algorithms base the decision on whether to increase the approximation order or to refine the mesh on an estimate of the local Sobolev regularity. Estimating explicitly the local Sobolev regularity is the basis of [1, 2, 3], [6], and more recently [17]. The approach of [17] generalises an idea proposed in [19], namely, to extract the regularity of the solution from the decay of the Legendre coefficients of the solution $u$. The work [19, 17] concentrates on one-dimensional problems and situations where all elements have tensor product structure (quadrilaterals, hexahedra); we extend this approach to triangulations consisting of triangles (in 2D) or tetrahedra (in 3D). We justify our procedure in Proposition 4.6 and Theorem 5.7 by showing that a function is analytic on the closure of a triangle/tetrahedron if and only if the coefficients of its expansion in orthogonal polynomials decay exponentially. Since the exact solution is not available, our numerical algorithm tests for exponential decay of the coefficients of the numerical solution by fitting them to an exponential decay law. The numerical studies of this paper show that an $h p$-adaptive algorithm based on testing locally for analyticity in this way works well provided that the initial polynomial degree is suitably large so that sufficiently many coefficients of the orthogonal expansion are available.

A more implicit way of gauging the regularity of a solution underlies the algorithms of [22] and [15]. In these algorithms the solution is assumed to be smooth and it is checked after a refinement step whether the assumption of smoothness is justified; if so, then a $p$ - 
enrichment is called for in the next step, otherwise an $h$-refinement will be performed. The algorithm proposed in [22] is studied there for meshes consisting of quadrilaterals only and mesh refinement is facilitated by hanging nodes. The present work extends this algorithm to the case of meshes consisting of triangles.

Another adaptive strategy that we study numerically in the present paper is the application of the "three-fold" algorithm of [14].

We close this introduction by briefly mentioning related ideas for $h p$-adaptivity. The approach pursued in $[8,9]$ consists in formulating $h p$-adaptivity as an optimization problem of finding the most efficient combination of $h$-refinement and $p$-enrichment. Earlier work consists of the Texas Three Step of [24, 23] and [7].

\section{Model problem and $h p$-FEM}

For a bounded Lipschitz domain $\Omega \subset \mathbb{R}^{2}$ and $f \in L^{2}(\Omega)$ we consider the following Dirichlet problem, given in weak formulation:

Problem 2.1. (model problem) Find $u \in H_{0}^{1}(\Omega)$ such that

$$
\int_{\Omega} \nabla u \cdot \nabla v d \Omega=\int_{\Omega} f v d \Omega \quad \forall v \in H_{0}^{1}(\Omega) .
$$

We will restrict our considerations to $\gamma$-shape-regular triangulations $\mathcal{T}$ of $\Omega$ consisting of affine triangles. That is, each element $K \in \mathcal{T}$ is the image $F_{K}(\hat{K})$ of the reference triangle $\hat{K}$, and we have

$$
h_{K}^{-1}\left\|F_{K}^{\prime}\right\|_{L^{\infty}(K)}+h_{K}\left\|\left(F_{K}^{\prime}\right)^{-1}\right\|_{L^{\infty}(K)} \leq \gamma \quad \forall K \in \mathcal{T}
$$

where $h_{K}$ denotes the diameter of the element $K$. In order to define $h p$-FEM spaces on a mesh $\mathcal{T}$, we associate a polynomial degree $p_{K} \in \mathbb{N}$ with each element $K \in \mathcal{T}$ and collect these $p_{K}$ in the polynomial degree vector $\mathbf{p}:=\left(p_{K}\right)_{K \in \mathcal{T}}$. We furthermore associate with each edge $e$ of the triangulation a polynomial degree

$$
p_{e}:=\min \left\{p_{K} \mid \text { e is an edge of element } K\right\}
$$

and denote by

$$
\mathbf{p}(K):=\left(p_{e 1}, p_{e 2}, p_{e 3}, p_{K}\right)
$$

the vector containing the polynomial distribution of the triangle $K \in \mathcal{T}$ with edges $\left\{e_{i} \mid i=\right.$ $1,2,3\}$. Next, we introduce the reference triangle $\hat{K}$ and the reference square $\hat{Q}$ by

$$
\hat{K}:=\{(x, y) \mid-1<x, y \wedge x+y<0\}, \quad \hat{Q}:=(-1,1)^{2}
$$

and point out the following relationship between these reference elements: 
Figure 1: Reference elements $\hat{K}$ and $\hat{Q}$
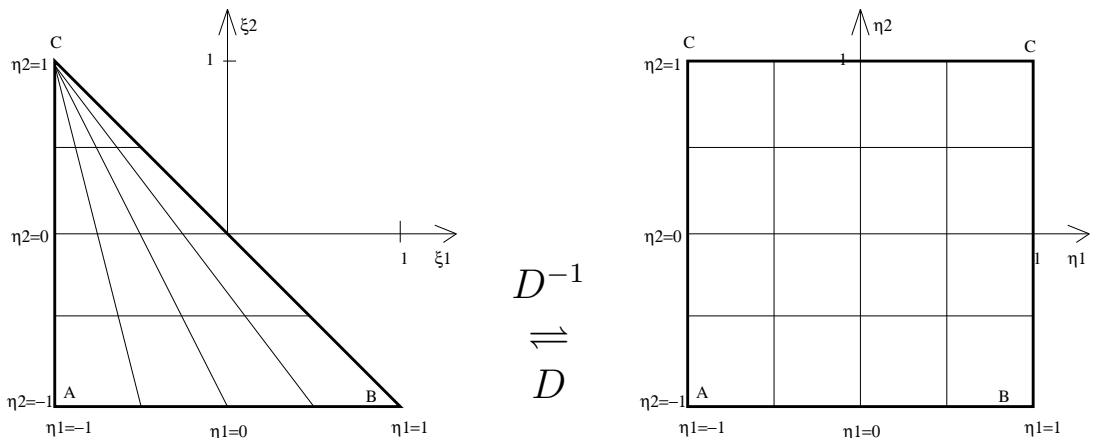

Lemma 2.2. (Duffy transformation) The transformation $D: \mathbb{R}^{2} \rightarrow \mathbb{R}^{2}$ given by

$$
D:\left(\eta_{1}, \eta_{2}\right) \mapsto\left(\xi_{1}, \xi_{2}\right)=\left(\frac{1}{2}\left(1+\eta_{1}\right)\left(1-\eta_{2}\right)-1, \eta_{2}\right)
$$

maps $\hat{Q}$ onto $\hat{K}$. The inverse transformation $D^{-1}: \mathbb{R}^{2} \rightarrow \mathbb{R}^{2}$ is given by

$$
D^{-1}:\left(\xi_{1}, \xi_{2}\right) \mapsto\left(\eta_{1}, \eta_{2}\right)=\left(2 \frac{1+\xi_{1}}{1-\xi_{2}}-1, \xi_{2}\right)
$$

Now we are in the position to define local shape functions on the reference triangle $\hat{K}$. To do so, we proceed as follows:

1. We define a set $\Phi$ of functions on the reference square $\hat{Q}$.

2. We transform these functions via $D: \mathbb{R}^{2} \rightarrow \mathbb{R}^{2}$ given by Lemma 2.2 to functions on the reference triangle $\hat{K}$.

Definition 2.3 (Local shape functions on the reference triangle). Let the reference elements $\hat{K}, \hat{Q}$ be given by (4) and let the transformation $D: \mathbb{R}^{2} \rightarrow \mathbb{R}^{2}$ be given by Lemma 2.2. Then, for a degree vector $\mathbf{p}(\hat{K})=\left(p_{A B}, p_{A C}, p_{B C}, p_{K}\right)$, where $p_{A B}, \ldots, p_{B C}$ denote the polynomial degrees associated with the edges $A B, \ldots, B C$ and $p_{K}$ denotes the polynomial degree associated with the interior of the triangle, we define a set of local shape functions as follows:

$$
\Psi^{B}:=\Phi^{B} \circ D^{-1}=\left\{\phi \circ D^{-1} \mid \phi \in \Phi^{B}\right\} \quad B=1, \ldots, 5,
$$


with $\Phi^{B}$ given by:

$$
\begin{aligned}
\Phi^{1} & =\left\{\frac{\left(1-\eta_{1}\right)}{2} \frac{\left(1-\eta_{2}\right)}{2}, \frac{\left(1+\eta_{1}\right)}{2} \frac{\left(1-\eta_{2}\right)}{2}, \frac{\left(1+\eta_{2}\right)}{2}\right\}, \\
\Phi^{2} & =\left\{\frac{\left(1-\eta_{1}\right)}{2} \frac{\left(1+\eta_{1}\right)}{2} \frac{\left(1-\eta_{2}\right)^{2}}{4} P_{i-1}^{(1,1)}\left(\eta_{1}\right) \mid i=1, \ldots, p_{A B}-1\right\}, \\
\Phi^{3} & =\left\{\frac{\left(1-\eta_{1}\right)}{2} \frac{\left(1-\eta_{2}\right)}{2} \frac{\left(1+\eta_{2}\right)}{2} P_{j-1}^{(1,1)}\left(\eta_{2}\right) \mid j=1, \ldots, p_{A C}-1\right\}, \\
\Phi^{4} & =\left\{\frac{\left(1+\eta_{1}\right)}{2} \frac{\left(1-\eta_{2}\right)}{2} \frac{\left(1+\eta_{2}\right)}{2} P_{j-1}^{(1,1)}\left(\eta_{2}\right) \mid j=1, \ldots, p_{B C}-1\right\}, \\
\Phi^{5} & =\left\{\frac{\left(1-\eta_{1}^{2}\right)}{4} \frac{\left(1+\eta_{2}\right)}{2}\left(\frac{1-\eta_{2}}{2}\right)^{i+1} P_{i-1}^{(1,1)}\left(\eta_{1}\right) P_{j-1}^{(2 i+1,1)}\left(\eta_{2}\right) \mid i, j=1, \ldots, p_{K}-1\right\}
\end{aligned}
$$

and $P_{i}^{(\alpha, \beta)}(\eta)$ the $i$-th Jacobi polynomial with respect to the weight $(1-\eta)^{\alpha}(1+\eta)^{\beta}$.

The subdivision of the shape functions into 5 different groups in Definition 2.3 follows a standard pattern in $h p$-FEM:

- $\Phi^{1}$ contains the vertex shape functions, which are the usual linear shape functions, equal to one in exactly one node and zero in the other nodes.

- The sets $\Phi^{2}, \Phi^{3}, \Phi^{4}$ contain the side shape functions, which are zero in all nodes and vanish on all but one edge.

- The set $\Phi^{5}$ consists of internal shape functions, which vanish on $\partial \hat{K}$.

Properties of this set of shape functions are collected in the following lemma.

Lemma 2.4. For $B=1, \ldots, 5$ let $\Psi^{B}$ be given by Definition 2.3. Denote by

$$
\mathcal{P}_{p}(\hat{K})=\operatorname{span}\left\{x^{i} y^{j} \mid 0 \leq i+j \leq p\right\}, \quad \mathcal{P}_{p}(I)=\operatorname{span}\left\{x^{i} \mid 0 \leq i \leq p\right\}
$$

the spaces of all polynomials of degree $p$ and set

$$
\begin{aligned}
& \mathcal{P}_{\mathbf{p}(\hat{\mathbf{K}})}(\hat{K})=\left\{\psi \in \mathcal{P}_{p_{K}}(\hat{K})|\psi|_{e_{i}} \in \mathcal{P}_{p_{e_{i}}}\left(e_{i}\right), i=1, \ldots, 3\right\}, \\
& \tilde{\mathcal{Q}}_{\mathbf{p}(\hat{K})}(\hat{K})=\operatorname{span} \Psi:=\operatorname{span}\left\{\bigcup_{B=1}^{5} \Psi^{B}\right\},
\end{aligned}
$$

where $e_{i}, i=1, \ldots, 3$ denote the edges of $\hat{K}$. Then

1. $\Psi:=\bigcup_{B=1}^{5} \Psi^{B}$ is a set of linear independent functions,

2. $\mathcal{P}_{\mathbf{p}(\hat{\mathbf{K}})}(\hat{K}) \subset \tilde{\mathcal{Q}}_{\mathbf{p}(\hat{K})}(\hat{K})$,

3. $\psi \in \Psi$ is polynomial. 
Proof. Obviously, the functions $\psi \in \Psi$ are independent. Since the shape functions defined in [18, D.1.1.2] are a subset of $\Psi$, the second claim follows from [18]. Moreover, in [18] it is already shown that for $B=1, \ldots, 4$ all functions $\psi \in \Psi^{B}$ are polynomial. Thus, it remains to show that $\phi \circ D^{-1}$ is polynomial for all $\phi \in \Phi^{5}$. Applying the transformation $D^{-1}$ to $\phi \in \Phi^{5}$ yields:

$$
\begin{aligned}
\phi \circ D^{-1}= & \left(\frac{-\xi_{1}-\xi_{2}}{1-\xi_{2}}\right)\left(\frac{1+\xi_{1}}{1-\xi_{2}}\right)\left(\frac{1+\xi_{2}}{2}\right)\left(\frac{1-\xi_{2}}{2}\right)^{(i+1)} \times \\
& P_{i-1}^{(1,1)}\left(2 \frac{1+\xi_{1}}{1-\xi_{2}}-1\right) P_{j-1}^{(2 i+1,1)}\left(\xi_{2}\right) .
\end{aligned}
$$

Now, by expanding the polynomials $P_{i-1}^{(1,1)}(2 x-1)=\sum_{k=0}^{i-1} a_{k} x^{k}$ and $P_{j-1}^{(2 i+1,1)}(x)=\sum_{k=0}^{j-1} b_{k} x^{k}$ we obtain

$$
\phi \circ D^{-1}=C_{i, j}\left(\xi_{1}+\xi_{2}\right)\left(1+\xi_{1}\right)\left(1+\xi_{2}\right)\left(1-\xi_{2}\right)^{(i-1)} \sum_{k=0}^{i-1} a_{k}\left(\frac{1+\xi_{1}}{1-\xi_{2}}\right)^{k} \sum_{k=0}^{j-1} b_{k} \xi_{2}^{k},
$$

which is polynomial in $\left(\xi_{1}, \xi_{2}\right)$.

Remark 2.5. The vertex and side shape functions introduced in Def. 2.3 coincide with those proposed by G. Karniadakis and S. Sherwin, [18]. The internal shape functions $\Phi^{5}$ differ from those of [18] in that we admit roughly twice as many internal shape functions. Our reason for using this bigger space is that the shape functions of $\Phi^{5}$ can be modified so as to be adapted to the quadrature rule employed. The stiffness matrix can then be set up in optimal complexity. These ideas have been presented for reference elements with tensor product structure (squares, hexahedra) in [21] and can be generalised to the present situation of triangles and tetrahedra, [12]. We emphasise that the additional shape functions are all internal shape functions that can be eliminated on the element level by static condensation.

Our $h p$-FEM spaces we define as follows:

Definition 2.6. (FEM spaces) Let $\mathcal{T}$ be a mesh consisting of triangles and let $\mathbf{p}$ be a polynomial degree vector. Furthermore, for all edges e let $p_{e}$ be given by (2). Then we set

$$
\begin{aligned}
& S^{\mathbf{p}}(\Omega, \mathcal{T}):=\left\{u \in H^{1}(\Omega) \mid u \circ F_{K} \in \tilde{\mathcal{Q}}_{\mathbf{p}(K)}(\hat{K}) \quad \forall K \in \mathcal{T}\right\}, \\
& S_{0}^{\mathbf{p}}(\Omega, \mathcal{T}):=S^{\mathbf{p}}(\Omega, \mathcal{T}) \cap H_{0}^{1}(\Omega),
\end{aligned}
$$

with $\tilde{\mathcal{Q}}_{\mathbf{p}(K)}(\hat{K})$ defined in Lemma 2.4.

The FE-discretization of Problem 2.1 then reads:

Problem 2.7. (hp-FEM approximation) Find $u_{h} \in S_{0}^{\mathbf{p}}(\Omega, \mathcal{T})$ such that

$$
\int_{\Omega} \nabla u \cdot \nabla v d \Omega=\int_{\Omega} f v d \Omega \quad \forall v \in S_{0}^{\mathbf{p}}(\Omega, \mathcal{T}) .
$$




\section{Error indicator}

This section is devoted to the residual-based a-posteriori error estimator used in the $h p$ adaptive algorithms of Section 4 to decide which elements to refine. The error estimator was developed in [22] and so we refer to [22] for a detailed description.

Definition 3.1 (error estimator). Let $K \in \mathcal{T}$. Then the local error indicator $\eta_{K}$, associated with the element $K$ is given by:

$$
\eta_{K}^{2}:=\eta_{B_{K}}^{2}+\eta_{E_{K}}^{2}
$$

where the first term $\eta_{B_{K}}^{2}$ is the weighted internal residual and the second term $\eta_{E_{K}}^{2}$ a weighted boundary residual. They are given by

$$
\eta_{B_{K}}^{2}:=\frac{h_{K}^{2}}{p_{K}^{2}}\left\|f_{p_{K}}+\Delta u_{F E}\right\|_{L^{2}(K)}^{2} \quad \text { and } \quad \eta_{E_{K}}^{2}:=\sum_{e \subset \partial K \cap \Omega} \frac{h_{e}}{2 p_{e}}\left\|\left[\frac{\partial u_{F E}}{\partial n_{e}}\right]\right\|_{L^{2}(e)}^{2},
$$

where $f_{K}$ denotes the $L^{2}(K)$-projection of $f$ on the space of polynomials of degree $p_{K}-1$ and $\left[\frac{\partial u_{F E}}{\partial n_{e}}\right]$ the jump of the normal derivative of $u_{F E}$ across the edge e. Finally, the global error indicator is given by

$$
\eta^{2}:=\sum_{K \in \mathcal{T}} \eta_{K}^{2}
$$

The following theorem collects the most important properties of the error indicator $\eta$ :

Theorem 3.2. Let $\epsilon>0$. Then there exist $C_{1}, C_{2}>0$ independent of $h$ and $\mathbf{p}$ such that

$$
\begin{gathered}
\left\|u-u_{F E}\right\|_{H^{1}(\Omega)}^{2} \leq C_{1} \sum_{K \in \mathcal{T}} \eta_{K}^{2}+\frac{h_{K}^{2}}{p_{K}^{2}}\left\|f-f_{p_{K}}\right\|_{L^{2}(K)}^{2}, \\
\eta_{K}^{2} \leq C_{2}(\epsilon) p_{K}^{1+2 \epsilon}\left(p_{K}\left\|u-u_{F E}\right\|_{H^{1}\left(\omega_{K}\right)}^{2}+p_{K}^{2 \epsilon} \frac{h_{K}^{2}}{p_{K}^{2}}\left\|f_{p_{K}}-f\right\|_{L^{2}\left(\omega_{K}\right)}^{2}\right) .
\end{gathered}
$$

\subsection{Performance of the error indicator}

In this subsection we present some numerical results to demonstrate the performance of the error indicator given by Definition 3.1. We introduce three examples:

Example 3.3. We consider the model problem (1) on $\Omega_{S}=(0,1)^{2}$ together with a right hand side $f$ chosen in such a way that the exact analytic solution is given by

$$
u=x(1-x) y(1-y)(1-2 y) e^{-\frac{5}{2}(2 x-1)^{2}} .
$$

Example 3.4. We consider the model problem (1) on $\Omega_{L}=(0,1)^{2} \backslash([0,1] \times[-1,0])$ together with a right-hand side $f$ chosen in such a way that the exact solution $u \in H^{5 / 3-\epsilon}\left(\Omega_{L}\right)$, $\epsilon>0$, is given by

$$
u=r^{\frac{2}{3}} \sin \left(\frac{2}{3} \varphi\right)\left(1-r^{2} \cos ^{2} \varphi\right)\left(1-r^{2} \sin ^{2} \varphi\right)
$$


Example 3.5. For $\Omega=(-1,1) \times(0,1), \Gamma_{N}=\{(x, y) \in \Omega \mid x>-0.4, y=0\}, \Gamma_{D}=\partial \Omega \backslash \Gamma_{N}$ and

$$
g_{N}(x, y)=\left\{\begin{array}{rll}
\frac{-1}{2 \sqrt{-x}} & : \quad x<0 \\
0 & : \quad x \geq 0
\end{array} \quad g(t)=\left\{\begin{aligned}
-a t^{3}-b t^{2}-c t-d & : t<-0.8 \\
1 & :|t| \leq 0.8 \\
a t^{3}-b t^{2}+c t-d & : t>0.8
\end{aligned}\right.\right.
$$

with $(a, b, c, d)=(250,675,600,175)$, we consider the problem: Find $u \in V:=\{u \in$ $H^{1}(\Omega)|u|_{\Gamma_{D}}=0$ such that

$$
\int_{\Omega} \nabla u \cdot \nabla v d \Omega=\int_{\Omega} f v d \Omega+\int_{\Gamma_{N}} g_{n} v d \Gamma \quad \forall v \in H_{0}^{1}(\Omega),
$$

where $f$ is chosen in such a way that the exact solution $u \in H^{3 / 2-\epsilon}(\Omega), \epsilon>0$, is given by

$$
u(r, \phi)=g(r \cos \phi) g(r \sin \phi) r^{1 / 2} \cos \left(\frac{\phi}{2}\right) .
$$

For a fixed polynomial degree $p$ the error indicator $\eta$ reduces to a standard $h$-FEM error indicator and therefore we are mainly interested in the $p$-dependence of $\eta$. In order to examine the $p$-dependence of $\eta$, we consider a pure $p$-FEM on a mesh consisting of 4 triangles in the case of Example 3.3, a mesh consisting of 12 triangles in the case of Example 3.4, and the mesh shown in Fig. 3 for Example 3.5. The important property of the mesh for Example 3.5 is that, in contrast to Example 3.4, the singularity is not at a mesh point. The results of our computation are plotted in Figures 2 and 3. All plots show the global error measured in the $H^{1}$-norm and the error predicted by $\eta$. As we can see, in each example the true error is overestimated by $\eta$ and especially in Example 3.4 we observe that as the polynomial degree $p$ increases, the true error decays much faster then the error indicator $\eta$ predicts. Note, however, that Theorem 3.2 is suboptimal in the efficiency estimate so that $\eta$ is allowed to overestimate the error. The situation of Example 3.4 is special: The solution is in some Sobolev space $H^{k}(\Omega)$, (here: $k=5 / 3-\varepsilon$ ), but the singularity is located at a mesh point; it is known from approximation theory that singularity functions of the type considered here can be approximated in the $H^{1}$ norm by polynomials of degree $p$ with an error $O\left(p^{-2(k-1)}\right)$; this convergence is faster than the $O\left(p^{-(k-1)}\right)$ behaviour achievable for generic functions in $H^{k}$, [5], [25, Sec. 3.3.5]. In Example 3.5, the singularity is not located at a mesh point. We observe in Fig. 3 and from the effectivity indices in Table 1 that in this situation the indicator $\eta$ captures the true error accurately. 
Figure 2: p-performance of the error indicator
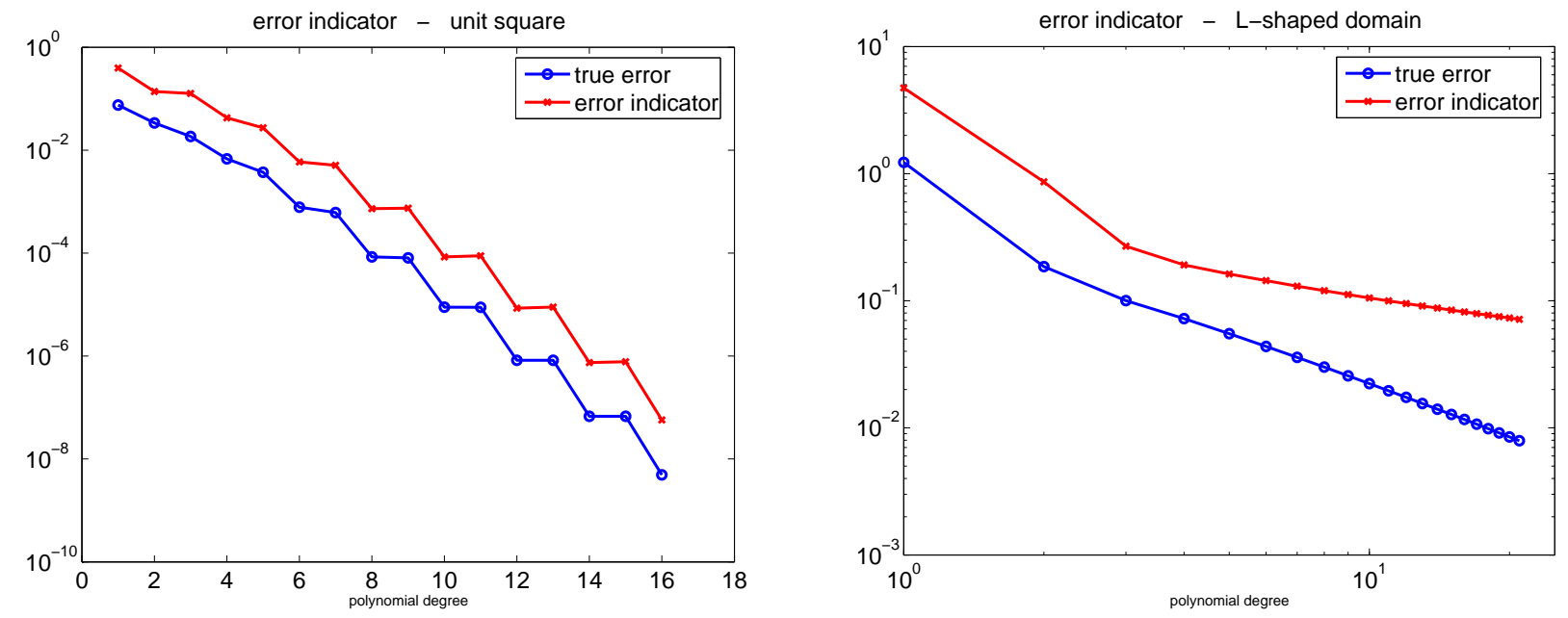

Figure 3: Example 3.5: mesh and p-performance of the error indicator
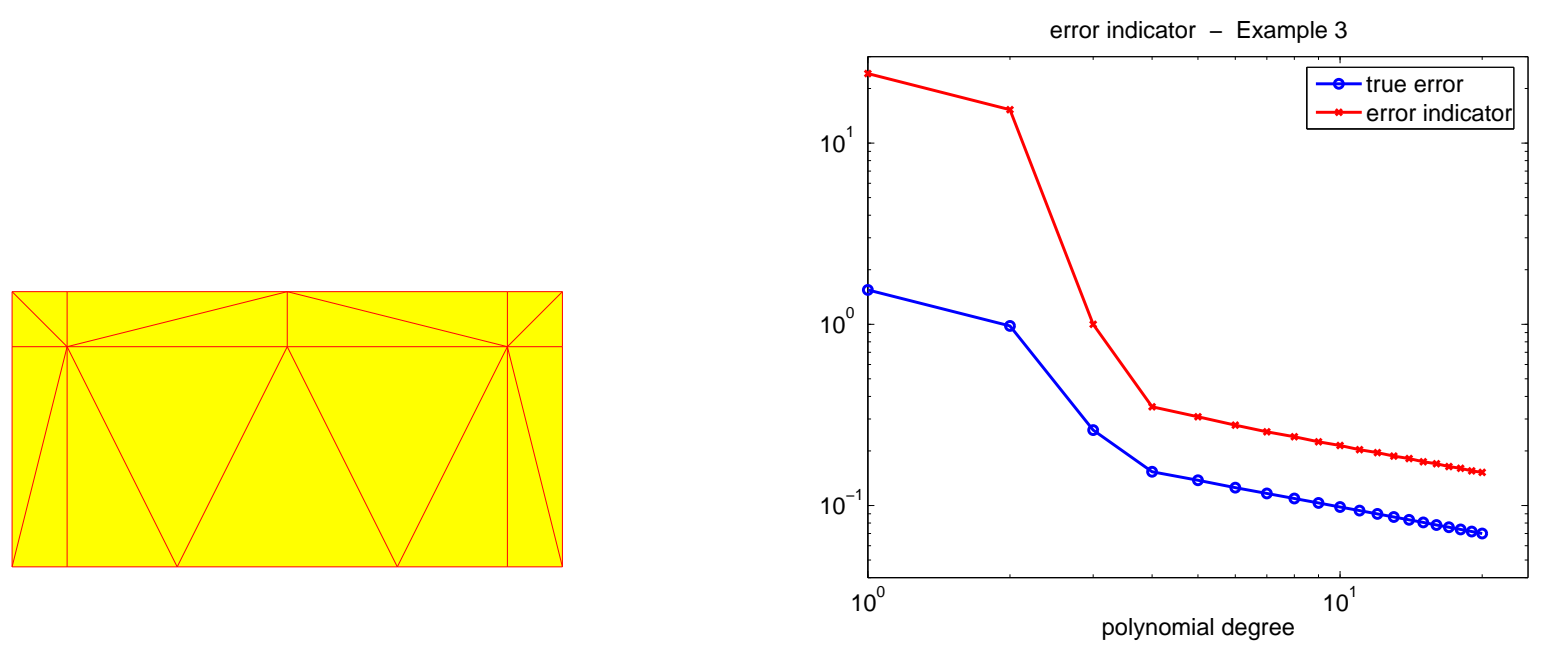
Table 1: effectivity index $\eta /\left\|u-u_{F E}\right\|_{H^{1}(\Omega)}$

\begin{tabular}{|r|rrr|r|rrr|}
\hline $\mathrm{p}$ & Ex. 1 & Ex. 2 & Ex. 3 & $\mathrm{p}$ & Ex. 1 & Ex. 2 & Ex. 3 \\
\hline 1 & 5.22 & 3.85 & 15.61 & 11 & 10.02 & 5.09 & 2.16 \\
2 & 4.08 & 4.64 & 15.60 & 12 & 10.27 & 5.47 & 2.18 \\
3 & 6.83 & 2.67 & 3.83 & 13 & 10.77 & 5.85 & 2.16 \\
4 & 6.24 & 2.64 & 2.28 & 14 & 11.02 & 6.24 & 2.17 \\
5 & 7.31 & 2.94 & 2.24 & 15 & 11.47 & 6.62 & 2.16 \\
6 & 7.62 & 3.29 & 2.21 & 16 & 11.62 & 7.02 & 2.17 \\
7 & 8.26 & 3.63 & 2.18 & 17 & 11.95 & 7.41 & 2.16 \\
8 & 8.66 & 3.99 & 2.19 & 18 & - & 7.81 & 2.17 \\
9 & 9.19 & 4.35 & 2.17 & 19 & - & 8.21 & 2.16 \\
10 & 9.49 & 4.72 & 2.18 & 20 & - & 8.61 & 2.17 \\
\hline
\end{tabular}

\section{$4 \quad h p$-adaptive strategies}

In this section we investigate and compare different $h p$-adaptive strategies. For each of these strategies we use the error indicator $\eta^{2}:=\sum_{K \in \mathcal{T}} \eta_{K}^{2}$ defined in Section 3 to determine which elements should be refined. Since the main difference between these strategies is the way how to decide whether to perform an $h$ - or a $p$ - refinement, we can formulate the following common basic $h p$-adaptive algorithm:

\section{Algorithm 4.1. (basic $h p$-adaptive algorithm)}

- Input: An admissible mesh $\mathcal{T}$ together with a polynomial degree distribution $\mathbf{p}:=$ $\left(p_{K}\right)_{K \in \mathcal{T}}$ and the corresponding finite element solution $u_{F E} \in S_{0}^{\mathbf{p}}(\Omega, \mathcal{T})$.

- Output: The refined mesh $\mathcal{T}_{\text {ref }}$ together with the polynomial degree distribution $\mathbf{p}:=$ $\left(p_{K}\right)_{K \in \mathcal{T}_{\text {ref }}}$.

- Algorithm:

1. Calculate the error indicator $\eta_{K}^{2}$ for all $K \in \mathcal{T}$.

2. Determine $\mathcal{T}_{\text {h_ref }} \subset \mathcal{T}$ containing all $K \in \mathcal{T}$ selected for h-refinement.

3. Determine $\mathcal{T}_{\text {p_ref }} \subset \mathcal{T}$ containing all $K \in \mathcal{T}$ selected for p-refinement.

4. Compute a preliminary version of the refined mesh $\mathcal{T}_{\text {ref }}$ by subdividing all $K \in$ $\mathcal{T}_{h_{-} \text {ref }}$ into four congruent sons (red refinement).

5. Determine the refined mesh $\mathcal{T}_{\text {ref }}$ by eliminating hanging nodes.

6. Increase the polynomial degree $p_{K}:=p_{K}+1$ for all elements $K \in \mathcal{T}_{\text {ref }} \cap \mathcal{T}_{p_{-} \text {ref }}$. (Otherwise, if an h-step is applied inherit the polynomial degree $p_{K}$ from the father element to the sons and leave it as it is if no refinement is applied.) 
Algorithm 4.2. (elimination of hanging nodes) The elimination of hanging nodes is done as follows:

1. While there exists an element $K \in \mathcal{T}_{h_{-} r e f}$ with more than one hanging node subdivide this element into four congruent sons (red refinement).

2. Subdivide all elements $K \in \mathcal{T}_{h-r e f}$ with one hanging node into two sons (green refinement).

Remark 4.3. Since green refinement divides an interior angles of the father element into two angles of about half the size of the original angle, repeated use of green refinement may lead to a degeneration of the mesh. In order to avoid such a degeneration, we forbid further refinement of triangles resulting from a green refinement. Instead of subdividing a so called green triangle, we undo the green refinement and perform a red subdivision of the father element before further refinement may occur. (For further information about adaptive mesh refinement strategies see [13] and the references therein.)

Now, having presented the basic algorithm, we consider different strategies for determining $\mathcal{T}_{h_{\text {rref }}}$, the set of all triangles selected for $h$-refinement, and $\mathcal{T}_{p_{-} \text {ref }}$, the set of all triangles selected for $p$-refinement.

\subsection{Strategy I - Comparison of estimated and predicted error}

The first strategy we consider is the strategy proposed in [22]. The decision which elements should be refined is based on a mean value strategy and to determine whether an $h$ refinement or a $p$-refinement is the proper one, we compare the current estimated error with the error predicted from a previous refinement step. Summarized this reads as follows:

Algorithm 4.4. For parameters $\sigma, \gamma_{h}, \gamma_{p}$ and $\gamma_{n}$ do:

1. Compute the mean error

$$
\bar{\eta}^{2}=\frac{1}{\# \mathcal{T}} \sum_{K \in \mathcal{T}} \eta_{K}^{2}
$$

2. Determine

$$
\begin{aligned}
& \mathcal{T}_{p_{-} \text {ref }}=\left\{K \in \mathcal{T} \mid \eta_{K}^{2} \geq \sigma \bar{\eta}^{2} \wedge \eta_{K}^{2}<\left(\eta_{K}^{\text {pred }}\right)^{2}\right\}, \\
& \mathcal{T}_{h_{\text {rref }}}=\left\{K \in \mathcal{T} \mid \eta_{K}^{2} \geq \sigma \bar{\eta}^{2} \wedge \eta_{K}^{2} \geq\left(\eta_{K}^{\text {pred }}\right)^{2}\right\} .
\end{aligned}
$$

For the predicted error of the initial triangulation we set

$$
\eta_{K}^{\text {pred }}=\left\{\begin{aligned}
0 & \text { if we prefer } h \text {-refinement for the first refinement step } \\
\infty & \text { if we prefer } p \text {-refinement for the first refinement step }
\end{aligned}\right.
$$

and after each adaptive refinement step, that is after finishing point 6 of the basic adaptive algorithm, we update the error prediction via the following algorithm: 
Algorithm 4.5 (error prediction). For all $K \in \mathcal{T}$ do

- If $K$ is h-refined, then for all $K_{s}=$ son of $K$ set

$$
\left(\eta_{K_{s}}^{\text {pred }}\right)^{2}:=\gamma_{h}\left(\eta_{K}^{\text {pred }}\right)^{2} \cdot\left\{\begin{array}{cl}
\frac{1}{4}\left(\frac{1}{2}\right)^{2 p_{K}} & \text { in the case of red subdivision } \\
\frac{1}{2} & \text { in the case of green subdivision }
\end{array} .\right.
$$

- If $K$ is p-refined, then

$$
\left(\eta_{K}^{\text {pred }}\right)^{2}:=\gamma_{p}\left(\eta_{K}^{\text {pred }}\right)^{2}
$$

- If no refinement is done, then

$$
\left(\eta_{K}^{\text {pred }}\right)^{2}:=\gamma_{n}\left(\eta_{K}^{\text {pred }}\right)^{2}
$$

(See Subsection 4.4 for a proper choice of $\sigma, \gamma_{h}, \gamma_{p}$ and $\gamma_{n}$ )

For the computation of $\eta_{K}^{\text {pred }}$ we followed the lines of [22] in the event of a red subdivision or a $p$-refinement. In the event of green refinement we observe the triangle $K$ is subdivided into two triangles without significantly reducing the edge length or the diameter. Thus, we don't expect a considerable error reduction and merely assign half the error of $K$ to each of its sons. The motivation for the decision between $h$ - and $p$-refinement is the following: The predictions (5) and (6) assume maximal smoothness of the solution; in fact the prediction (6) assumes analyticity of the solution so that the error can decay exponentially. If the estimated error is smaller than the predicted error, then we perform $p$-refinement since the assumption of smoothness appears to be correct. Otherwise, if the estimated error is larger than the predicted error, we perform $h$-refinement since our assumption of smoothness does not appear to be correct.

\subsection{Strategy II - Decay of Legendre expansion coefficients}

The second second strategy we consider is to determine whether the solution is locally smooth or not by a expansion in orthogonal polynomials of the finite element solution. This idea was first discussed in [19] (see also [17]). The theoretical basis for the case of triangular elements is the following result:

Proposition 4.6. Define on the reference triangle $\hat{K}$ the $L^{2}(\hat{K})$-orthogonal basis $\psi_{p q}, p$, $q \in \mathbb{N}_{0}$ by

$$
\psi_{p q}=\tilde{\psi}_{p q} \circ D^{-1} \quad \text { with } \tilde{\psi}_{p q}=P_{p}^{(0,0)}\left(\eta_{1}\right)\left(\frac{1-\eta_{2}}{2}\right)^{p} P_{q}^{(2 p+1,0)}\left(\eta_{2}\right),
$$

where $P_{p}^{(\alpha, \beta)}(\eta)$ denotes the $p$-th Jacobi polynomial with respect to the weight $(1-\eta)^{\alpha}(1+\eta)^{\beta}$ and $D$ the transformation of Lemma 2.2. Let $u \in L^{2}(\hat{K})$ written as $u=\sum_{p, q \in \mathbb{N}_{0}} u_{p q} \psi_{p q}$. Then $u$ is analytic on $\overline{\hat{K}}$ if and only if there exist constants $C, b>0$ such that $\left|u_{p q}\right| \leq$ $C e^{-b(p+q)}$ for all $p, q \in \mathbb{N}_{0}$. 
Proof. Combine [20, Prop. 3.2.14] and [20, Lemma 3.2.15].

We will present the extension of Proposition 4.6 to tetrahedra below in Theorem 5.7. The main idea of an $h p$-algorithm that is based on estimating the decay of the coefficients is to check whether the expansion coefficients $u_{p q}$ of $\left(\left.u_{F E}\right|_{K}\right) \circ F_{K}=\sum_{p, q} u_{p q} \psi_{p q}$ decay sufficiently fast. If so, then an exponential convergence in $p$ can be expected and a $p$ refinement is indicated. Otherwise, an $h$-refinement is called for. Since the exact solution $\left.u\right|_{K}$ is not available, we will consider the expansion of the finite element approximation. This leads to:

Algorithm 4.7. For parameters $\sigma$ and $\delta$ do:

1. Compute the mean error

$$
\bar{\eta}^{2}=\frac{1}{\# \mathcal{T}} \sum_{K \in \mathcal{T}} \eta_{K}^{2}
$$

2. For all elements $K \in \mathcal{T}$ with $\eta_{K}^{2} \geq \sigma \bar{\eta}^{2}$ compute the expansion coefficients

$$
u_{i j ; K}=\left\|\psi_{i j}\right\|_{L^{2}(\hat{K})}^{-2}\left(\left.u_{F E}\right|_{K} \circ F_{K}^{-1}, \psi_{i j}\right)_{L^{2}(\hat{K})}, \quad 0 \leq i+j \leq p_{K}
$$

and estimate the decay coefficient $b_{K}$ by a least-squares fit of

$$
\ln \left|u_{i j ; K}\right| \sim C_{K}-b_{K}(i+j) .
$$

3. Determine

$$
\begin{aligned}
& \mathcal{T}_{\text {p_ref }}=\left\{K \in \mathcal{T} \mid \eta_{K}^{2} \geq \sigma \bar{\eta}^{2} \wedge b_{K} \geq \delta\right\} \\
& \mathcal{T}_{\text {h_ref }}=\left\{K \in \mathcal{T} \mid \eta_{K}^{2} \geq \sigma \bar{\eta}^{2} \wedge b_{K}<\delta\right\} .
\end{aligned}
$$

\subsection{Strategy III - Three fold algorithm}

The third strategy we consider goes back to an idea proposed in [14] for the treatment of hypersingular and weakly singular integral equations via boundary element method. In contrast to the previous strategies, the decision which elements should be refined is no longer based on a mean value strategy. Instead, the crucial value for refinement is the maximum occurring error. The main idea of the algorithm is quite simple: If the error indicator $\eta_{K}$ predicts a small error for $K \in \mathcal{T}$ (with respect to the maximum occurring error), we do nothing. For elements with medium predicted error we perform a $p$-enrichment and those elements with a large predicted error become $h$-refined. Thus, the algorithm reads as follows:

Algorithm 4.8. For parameters $\delta_{1}, \delta_{2}$ with $0<\delta_{1}<\delta_{2}<1$ do:

1. Compute the maximum error

$$
\eta_{\max }^{2}=\max _{K \in \mathcal{T}} \eta_{K}^{2}
$$

2. Determine

$$
\begin{aligned}
\mathcal{T}_{p_{-} \text {ref }} & =\left\{K \in \mathcal{T} \mid \delta_{1} \eta_{\text {max }}^{2} \leq \eta_{K}^{2} \leq \delta_{2} \eta_{\text {max }}^{2}\right\} \\
\mathcal{T}_{h_{-} \text {ref }} & =\left\{K \in \mathcal{T} \mid \eta_{K}^{2}>\delta_{2} \eta_{\text {max }}^{2}\right\} .
\end{aligned}
$$




\subsection{Collection of numerical results}

In this section the performance of each adaptive $h p$-strategy from above is testest for two very different problems. The first example we consider is Example 3.3. Since we have an analytic solution, the optimal strategy is a pure $p$-method on a suitable mesh, which features an exponential convergence in the polynomial degree $p$. We therefore expect a successful $h p$-algorithm to perform only a few $h$-refinements at the beginning and then turn into a pure $p$-method in later iterations. Anticipating an exponential convergence in $p$ we plot the error versus $\sqrt{\mathrm{DOF}}$. The second example we consider is Example 3.4, the classical L-shaped domain. In this case we have a singularity at the origin and so we expect a strong mesh refinement towards this reentrant corner in conjunction with $p$-refinement for the rest of the domain. For this example, the best known $h p$-strategy yields an error bound of

$$
\left\|u-u_{F E}\right\|_{H^{1}(\Omega)} \leq C e^{-b(\mathrm{DOF})^{1 / 3}}
$$

(see, e.g., [25]); for this example, therefore, we plot the error versus (DOF) $)^{1 / 3}$. All computations are performed with the $h p$-FEM code ADURACON ${ }^{1}$. We now consider the strategies in detail:

- Strategy I: For our computations we choose: $\sigma=0.75, \gamma_{p}=0.7, \gamma_{h}=4.0$ and $\gamma_{n}=1.0$ together with $\eta_{K}^{\text {pred }}=0$ for all $K \in \mathcal{T}$. The corresponding results are shown in Tables 2, 3 and Figures 4-6, 13, 16, 19. As we observe, Strategy I performs well in both cases. In the case of Example 3.3 we obtain the expected p-method after a few mesh refinement steps; Example 3.4 features a strong mesh refinement towards the singularity at the reentrant corner and $p$-refinement in the remainder of the domain where the solution is smooth. In comparison to the other strategies, we obtain a slightly increased number of $h$-refinements and relatively large polynomial degree of $p_{K} \in\{4 ; 5\}$ for the elements at the reentrant corner. A possible reason for the increasing number of $h$-refinements in Table 2 at higher iteration levels could be limitations of computational accuracy. In such a case the error does not decrease further and consequently the algorithm always suggests $h$-refinement.

- Strategy II: The computations are based on: $\sigma=0.75, \delta=1.0$. Moreover, in order to obtain a sufficing number of Legendre expansion coefficients, which is necessary to achieve a good estimation for the decay coefficient $b$, we start with an initial polynomial degree distribution $p_{K}=3$ for all $K \in \mathcal{T}$. As we can see (Table 4 , Figure 7 and following), for both cases, Example 3.3 and Example 3.4, the algorithm performs well. In Example 3.3 the error appears to have reached the limit of computational accuracy after 18 iterations.

- Strategy III: For our computations we choose: $\delta_{1}=0.07, \delta_{2}=0.7$. We observe that in the case of Example 3.4 the algorithm performs well but it performs a considerable number of $h$-refinements for the analytic solution on the unit square. However, this

\footnotetext{
${ }^{1}$ see [11]
} 
"failure" is predictable: If we consider Algorithm 4.8, we observe that the algorithm is not designed for problems whose solution is smooth on the whole domain. In fact, it is impossible to achieve pure $p$-enrichment since for nearly uniform error distributions the algorithm opts for a pure $h$-refinement. This happened, for example, in Level 13.

Remark 4.9. Throughout all of our computations we observed that the majority of the wrong decisions concerning $h$ - or p-refinement, in particular an increasing of the polynomial degree in regions where the solution is not smooth, occur in the initial steps of the iteration. Wrong decisions in later stages are rather an exception. (See the mesh and polynomial degree distribution near the reentrant corner of the L-shaped domain.) We therefore expect an improvement by combining the strategies above with a proper coarsening algorithm that corrects excessive p-refinement in regions where the solution is not smooth. 
Figure 4: Strategy I - L-shaped domain - Iteration levels 0, 15, and 25
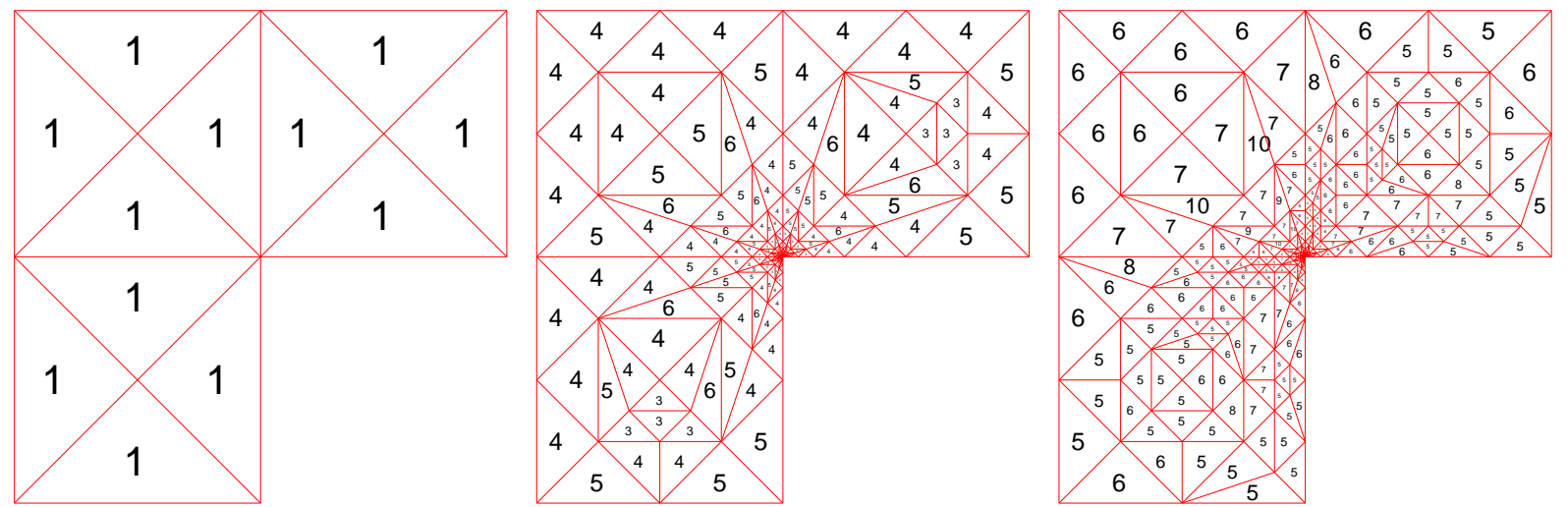

Figure 5: Zoom near reentrant corner: Iteration level 15 (magnification factor $2^{9}$ ) - Iteration level 25 (magnification factor $2^{19}$ )
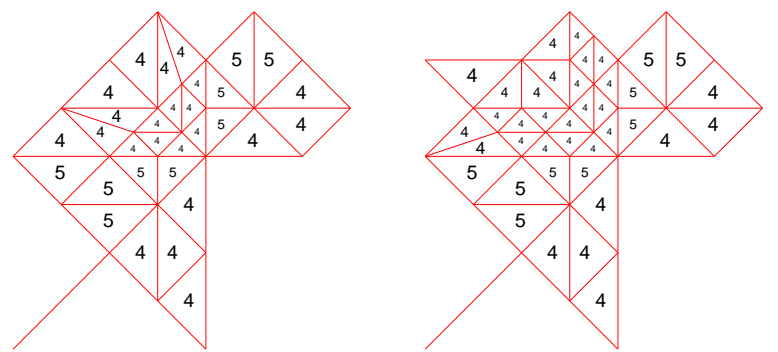

Figure 6: Strategy I - unit square - Iteration levels 0, 10, and 20

Table 2: Strategy I - L-shaped domain - Number of elements, maximum polynomial degree, $h$ - and $p$-refinements per level

\begin{tabular}{|c|cccc||c|cccc|}
\hline Level & $\# \mathcal{T}$ & $p_{\max }$ & $h_{\text {ref }}$ & $p_{\text {ref }}$ & Level & $\# \mathcal{T}$ & $p_{\max }$ & $h_{\text {ref }}$ & $p_{\text {ref }}$ \\
\hline 0 & 12 & 1 & 12 & 0 & 13 & 238 & 5 & 14 & 10 \\
1 & 40 & 1 & 8 & 26 & 14 & 262 & 6 & 14 & 20 \\
2 & 48 & 2 & 8 & 14 & 15 & 286 & 6 & 22 & 36 \\
3 & 60 & 3 & 0 & 26 & 16 & 318 & 7 & 14 & 32 \\
4 & 60 & 3 & 0 & 20 & 17 & 342 & 7 & 14 & 28 \\
5 & 60 & 4 & 6 & 16 & 18 & 366 & 7 & 14 & 34 \\
6 & 70 & 5 & 14 & 12 & 19 & 390 & 8 & 42 & 42 \\
7 & 94 & 5 & 14 & 8 & 20 & 442 & 8 & 34 & 50 \\
8 & 118 & 5 & 14 & 12 & 21 & 490 & 9 & 32 & 50 \\
9 & 142 & 5 & 14 & 20 & 22 & 536 & 10 & 18 & 54 \\
10 & 166 & 5 & 14 & 10 & 23 & 568 & 10 & 26 & 78 \\
11 & 190 & 5 & 14 & 14 & 24 & 612 & 10 & 26 & 80 \\
12 & 214 & 5 & 14 & 8 & 25 & 656 & 10 & 36 & 82 \\
\hline
\end{tabular}

Table 3: Strategy I - unit square - Number of elements, maximum polynomial degree, $h$ and $p$-refinements per level

\begin{tabular}{|c|cccc||c|cccc|}
\hline Level & $\# \mathcal{T}$ & $p_{\max }$ & $h_{\text {ref }}$ & $p_{\text {ref }}$ & Level & $\# \mathcal{T}$ & $p_{\max }$ & $h_{\text {ref }}$ & $p_{\text {ref }}$ \\
\hline 0 & 16 & 1 & 12 & 0 & 11 & 104 & 6 & 0 & 33 \\
1 & 40 & 1 & 12 & 14 & 12 & 104 & 6 & 0 & 35 \\
2 & 52 & 2 & 12 & 12 & 13 & 104 & 7 & 0 & 32 \\
3 & 64 & 2 & 16 & 23 & 14 & 104 & 8 & 0 & 41 \\
4 & 92 & 3 & 8 & 37 & 15 & 104 & 9 & 0 & 37 \\
5 & 104 & 3 & 0 & 50 & 16 & 104 & 9 & 0 & 49 \\
6 & 104 & 4 & 0 & 36 & 17 & 104 & 9 & 0 & 49 \\
7 & 104 & 4 & 0 & 34 & 18 & 104 & 10 & 0 & 37 \\
8 & 104 & 5 & 0 & 44 & 19 & 104 & 11 & 0 & 36 \\
9 & 104 & 5 & 0 & 42 & 20 & 104 & 12 & 0 & 42 \\
10 & 104 & 6 & 0 & 46 & & & & & \\
\hline
\end{tabular}


Figure 7: Strategy II - L-shaped domain - Iteration levels 0, 15, and 25
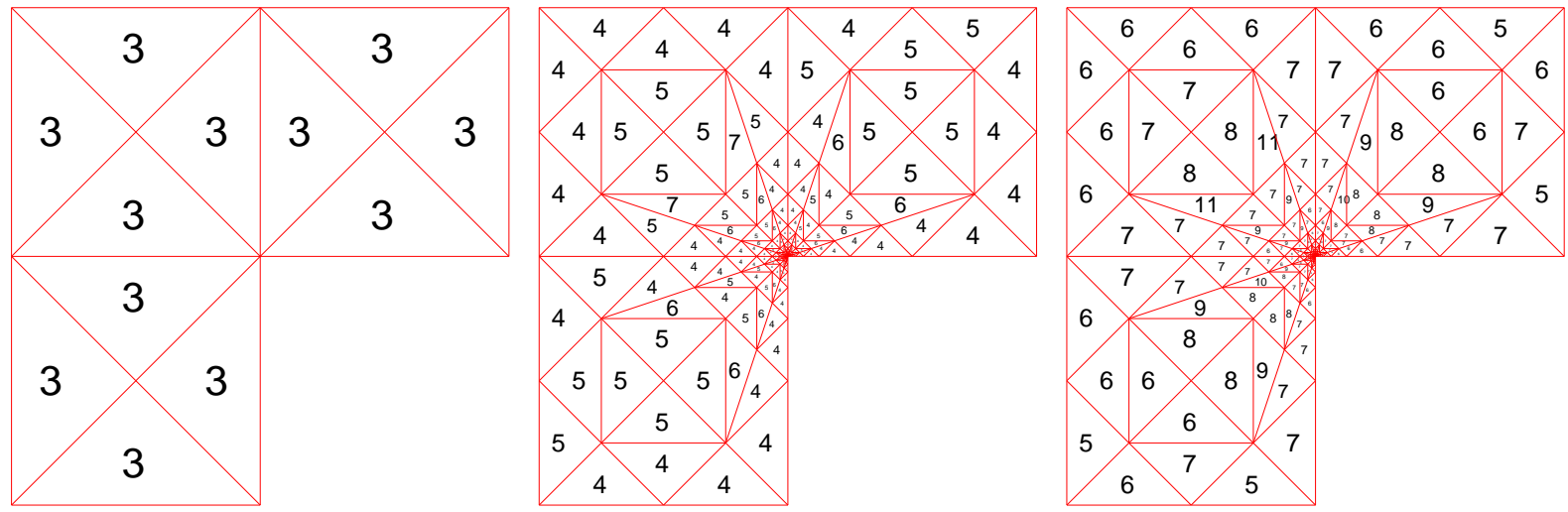

Figure 8: Zoom near reentrant corner - Iteration level 15 (magnification factor $2^{11}$ ) Iteration level 25 (magnification factor $2^{21}$ )


Figure 9: Strategy II - unit square - Iteration levels 0, 10 and 20

Table 4: Strategy II - L-shaped domain - Number of elements, maximum polynomial degree, $h$ - and $p$-refinements per level

\begin{tabular}{|c|cccc||c|cccc|}
\hline Level & $\# \mathcal{T}$ & $p_{\max }$ & $h_{\text {ref }}$ & $p_{\text {ref }}$ & Level & $\# \mathcal{T}$ & $p_{\max }$ & $h_{\text {ref }}$ & $p_{\text {ref }}$ \\
\hline 0 & 12 & 3 & 10 & 0 & 13 & 292 & 6 & 16 & 20 \\
1 & 30 & 3 & 10 & 4 & 14 & 316 & 6 & 16 & 28 \\
2 & 40 & 4 & 14 & 2 & 15 & 340 & 7 & 16 & 44 \\
3 & 58 & 4 & 12 & 0 & 16 & 364 & 7 & 16 & 42 \\
4 & 76 & 4 & 16 & 0 & 17 & 388 & 8 & 16 & 48 \\
5 & 100 & 4 & 16 & 0 & 18 & 412 & 8 & 16 & 48 \\
6 & 124 & 4 & 16 & 0 & 19 & 436 & 8 & 16 & 68 \\
7 & 148 & 4 & 16 & 14 & 20 & 460 & 9 & 16 & 68 \\
8 & 172 & 4 & 16 & 20 & 21 & 484 & 9 & 16 & 76 \\
9 & 196 & 4 & 16 & 10 & 22 & 508 & 9 & 16 & 80 \\
10 & 220 & 5 & 16 & 6 & 23 & 532 & 9 & 16 & 100 \\
11 & 244 & 5 & 16 & 2 & 24 & 556 & 10 & 16 & 92 \\
12 & 268 & 5 & 16 & 14 & 25 & 580 & 11 & 16 & 112 \\
\hline
\end{tabular}

Table 5: Strategy II - unit square - Number of elements, maximum polynomial degree, $h$ and $p$-refinements per level

\begin{tabular}{|c|cccc||c|cccc|}
\hline Level & $\# \mathcal{T}$ & $p_{\max }$ & $h_{\text {ref }}$ & $p_{\text {ref }}$ & Level & $\# \mathcal{T}$ & $p_{\max }$ & $h_{\text {ref }}$ & $p_{\text {ref }}$ \\
\hline 0 & 16 & 3 & 0 & 4 & 11 & 80 & 9 & 16 & 33 \\
1 & 16 & 4 & 8 & 4 & 12 & 104 & 9 & 0 & 28 \\
2 & 32 & 4 & 0 & 8 & 13 & 104 & 10 & 0 & 34 \\
3 & 32 & 5 & 0 & 16 & 14 & 104 & 10 & 0 & 42 \\
4 & 32 & 6 & 12 & 4 & 15 & 104 & 11 & 0 & 34 \\
5 & 56 & 6 & 0 & 16 & 16 & 104 & 11 & 0 & 26 \\
6 & 56 & 6 & 0 & 12 & 17 & 104 & 12 & 0 & 24 \\
7 & 56 & 7 & 0 & 34 & 18 & 104 & 13 & 0 & 43 \\
8 & 56 & 8 & 16 & 9 & 19 & 104 & 13 & 0 & 44 \\
9 & 80 & 8 & 0 & 28 & 20 & 104 & 13 & 0 & 47 \\
10 & 80 & 9 & 0 & 24 & & & & & \\
\hline
\end{tabular}


Figure 10: Strategy III - L-shaped domain - Iteration levels 0, 15, and 25
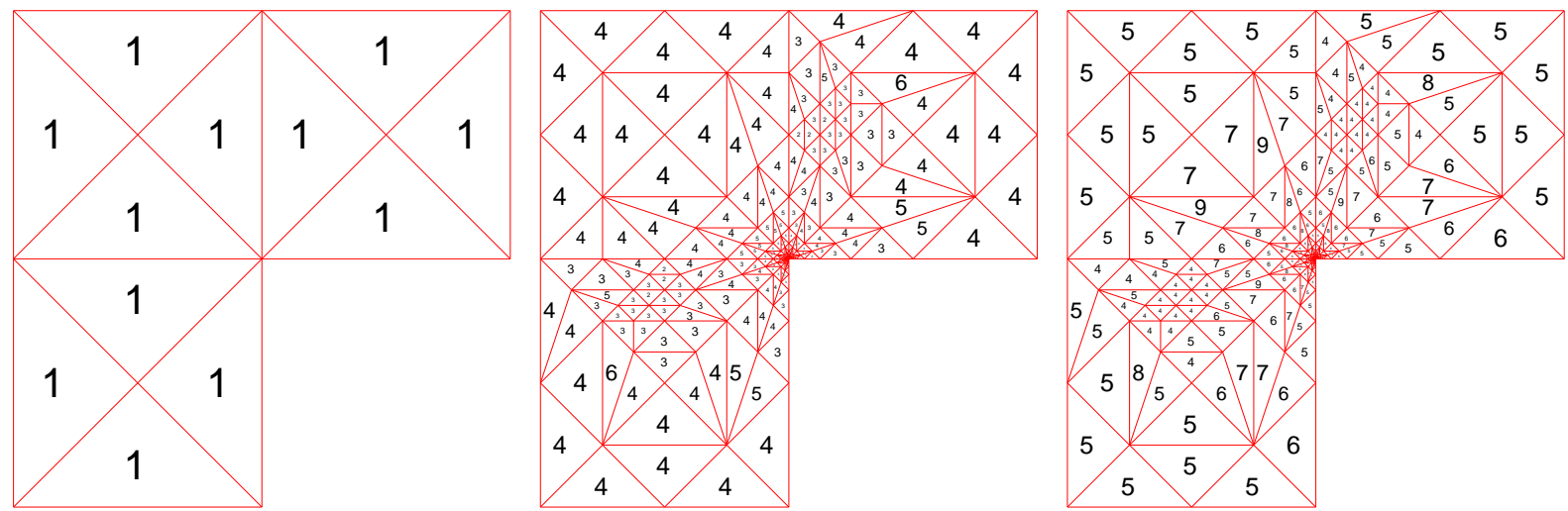

Figure 11: Zoom near reentrant corner - Iteration level 15 (magnification factor $2^{9}$ ) Iteration level 25 (magnification factor $2^{19}$ )
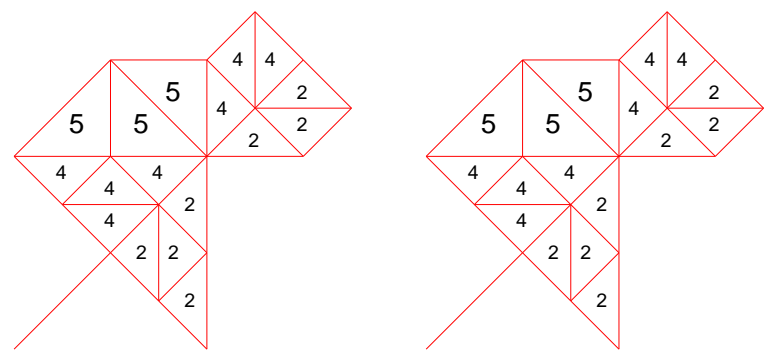

Figure 12: Strategy III - unit square - Iteration levels 0, 10, and 20
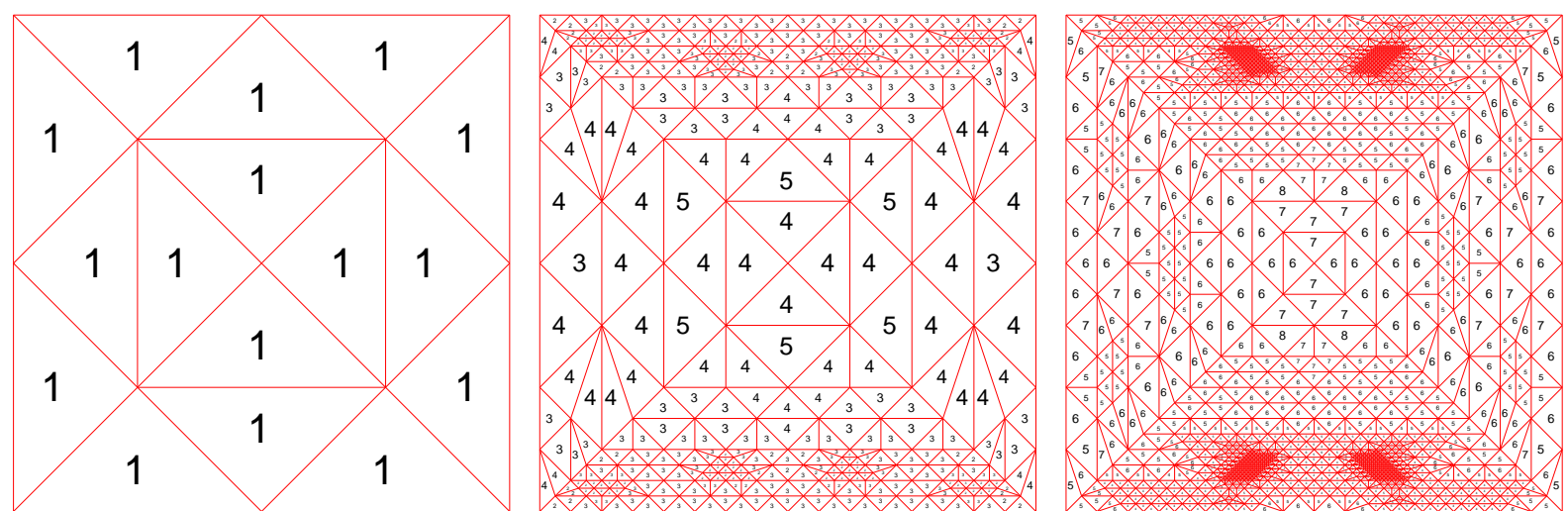
Table 6: Strategy III - L-shaped domain - Number of elements, maximum polynomial degree, $h$ - and $p$-refinements per level

\begin{tabular}{|c|cccc||c|cccc|}
\hline Level & $\# \mathcal{T}$ & $p_{\max }$ & $h_{\text {ref }}$ & $p_{\text {ref }}$ & Level & $\# \mathcal{T}$ & $p_{\max }$ & $h_{\text {ref }}$ & $p_{\text {ref }}$ \\
\hline 0 & 12 & 1 & 6 & 2 & 13 & 262 & 6 & 14 & 32 \\
1 & 26 & 2 & 8 & 18 & 14 & 286 & 6 & 14 & 36 \\
2 & 34 & 2 & 12 & 10 & 15 & 310 & 6 & 14 & 28 \\
3 & 52 & 3 & 16 & 18 & 16 & 334 & 6 & 14 & 46 \\
4 & 68 & 4 & 28 & 14 & 17 & 358 & 6 & 14 & 40 \\
5 & 112 & 4 & 6 & 42 & 18 & 382 & 7 & 14 & 44 \\
6 & 118 & 5 & 12 & 10 & 19 & 406 & 7 & 14 & 48 \\
7 & 136 & 5 & 6 & 2 & 20 & 430 & 8 & 14 & 40 \\
8 & 142 & 5 & 14 & 8 & 21 & 454 & 8 & 14 & 56 \\
9 & 166 & 5 & 14 & 18 & 22 & 478 & 8 & 14 & 82 \\
10 & 190 & 5 & 14 & 22 & 23 & 502 & 8 & 14 & 66 \\
11 & 214 & 5 & 14 & 32 & 24 & 526 & 8 & 14 & 82 \\
12 & 238 & 5 & 14 & 34 & 25 & 550 & 9 & 14 & 96 \\
\hline
\end{tabular}

Table 7: Strategy III - unit square - Number of elements, maximum polynomial degree, $h$ and $p$-refinements per level

\begin{tabular}{|c|cccc||c|cccc|}
\hline Level & $\# \mathcal{T}$ & $p_{\max }$ & $h_{\text {ref }}$ & $p_{\text {ref }}$ & Level & $\# \mathcal{T}$ & $p_{\max }$ & $h_{\text {ref }}$ & $p_{\text {ref }}$ \\
\hline 0 & 16 & 1 & 8 & 0 & 11 & 512 & 6 & 144 & 115 \\
1 & 28 & 1 & 12 & 12 & 12 & 768 & 7 & 48 & 135 \\
2 & 40 & 2 & 12 & 20 & 13 & 832 & 7 & 500 & 92 \\
3 & 64 & 3 & 44 & 12 & 14 & 1868 & 7 & 20 & 8 \\
4 & 132 & 3 & 68 & 44 & 15 & 1888 & 7 & 68 & 951 \\
5 & 240 & 3 & 64 & 104 & 16 & 1972 & 7 & 16 & 152 \\
6 & 340 & 4 & 56 & 104 & 17 & 2000 & 7 & 164 & 182 \\
7 & 408 & 4 & 28 & 48 & 18 & 2208 & 7 & 40 & 40 \\
8 & 444 & 4 & 16 & 36 & 19 & 2248 & 7 & 204 & 258 \\
9 & 460 & 4 & 16 & 202 & 20 & 2564 & 8 & 16 & 495 \\
10 & 476 & 5 & 24 & 132 & & & & & \\
\hline
\end{tabular}


Figure 13: Strategy I - Performance of $h p$-adaptive algorithm
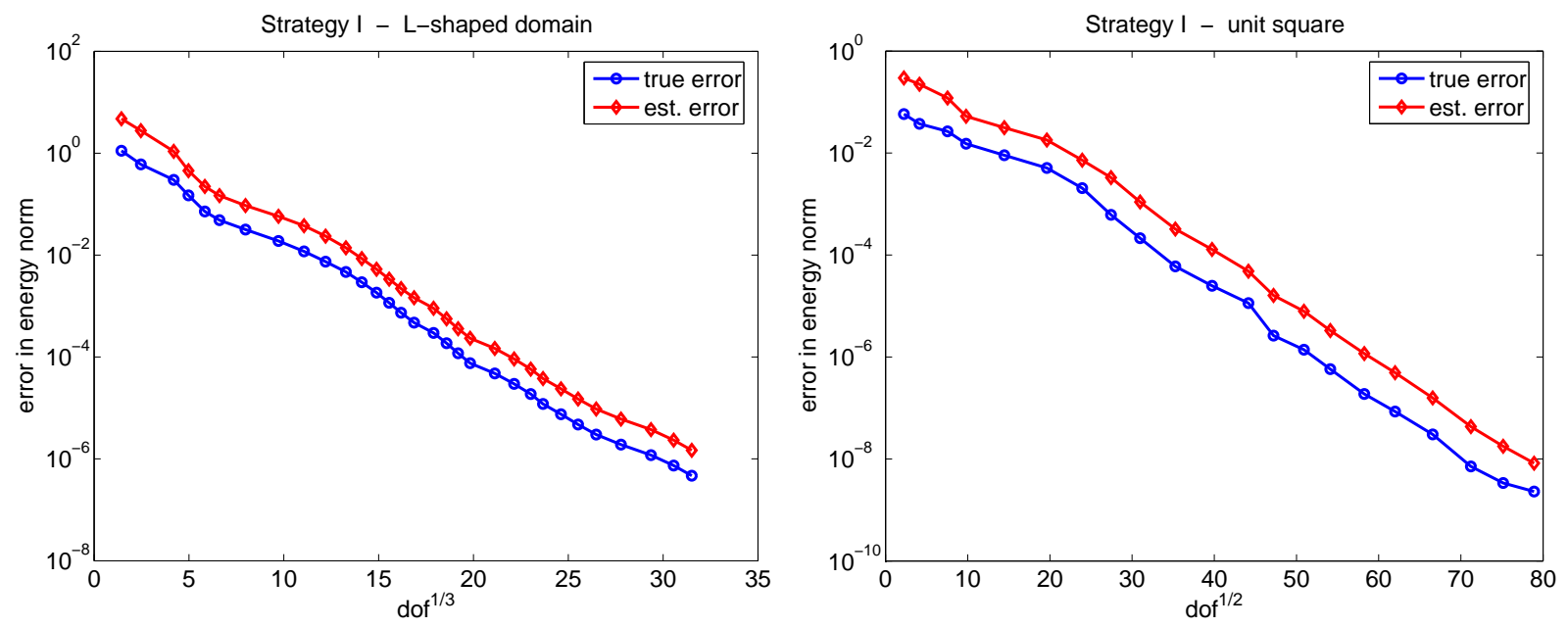

Figure 14: Strategy II - Performance of $h p$-adaptive algorithm


Figure 15: Strategy III - Performance of $h p$-adaptive algorithm
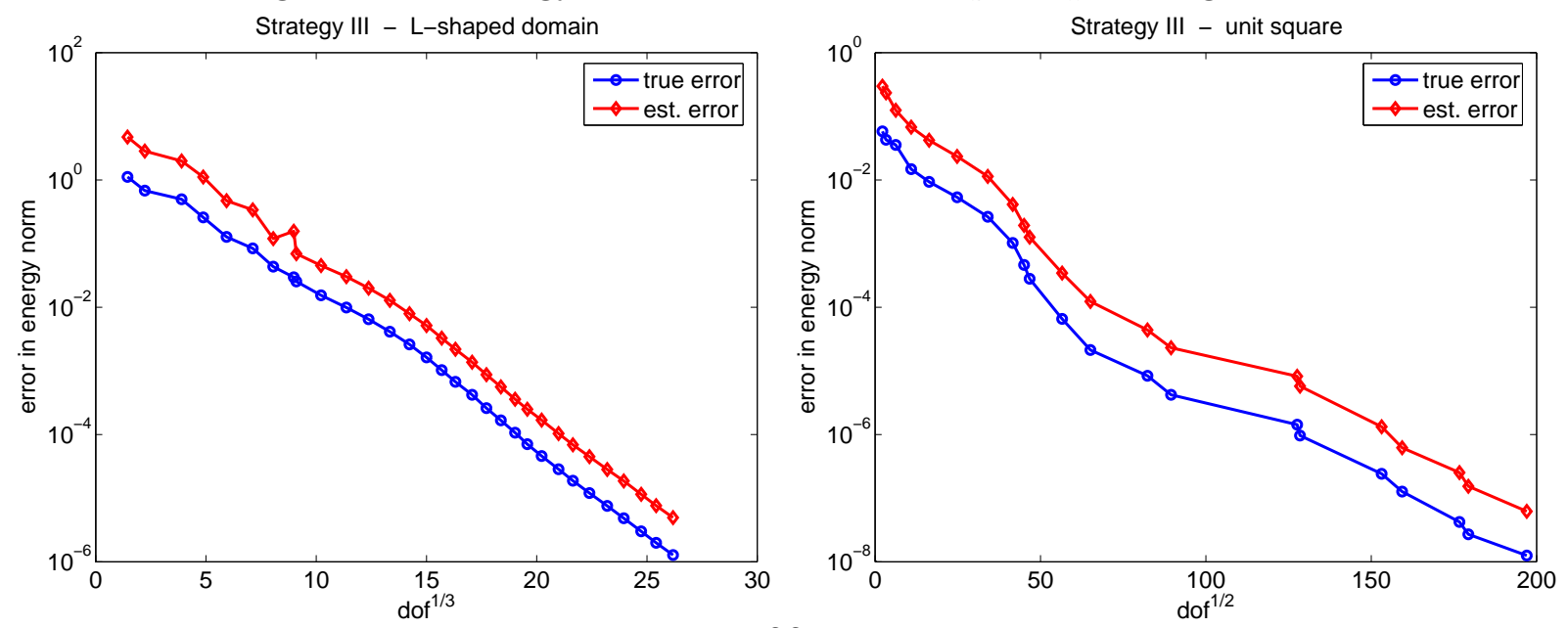
Figure 16: Strategy I - L-shaped domain - Polynomial degree distribution along the line from $(0,0)$ to $(-1 / 2,1)$ - Iteration levels 15 and 25
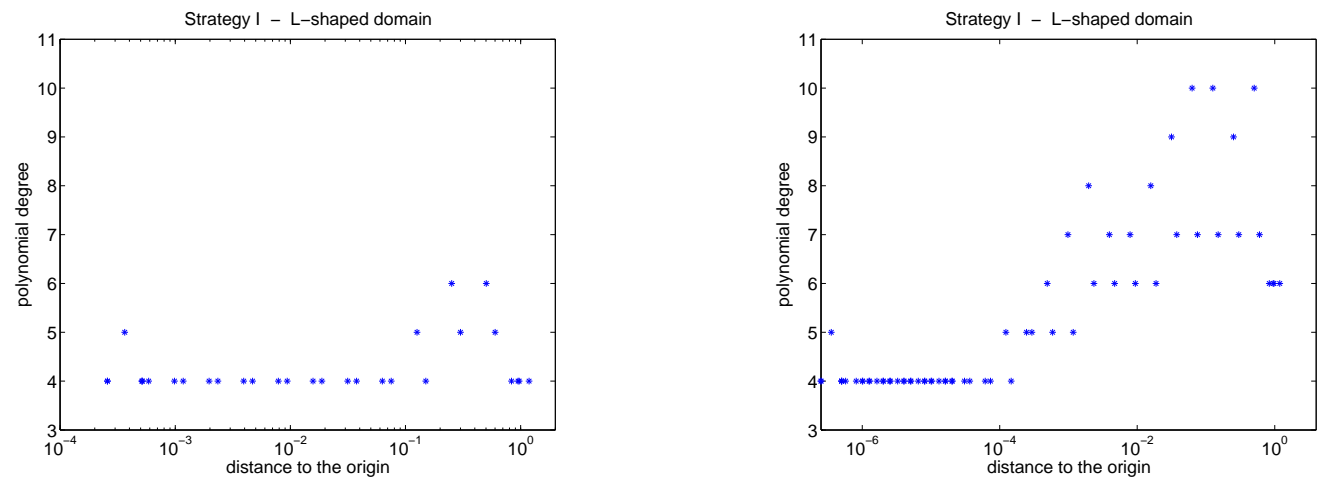

Figure 17: Strategy II - L-shaped domain - Polynomial degree distribution along the line from $(0,0)$ to $(-1 / 2,1)$ - Iteration levels 15 and 25
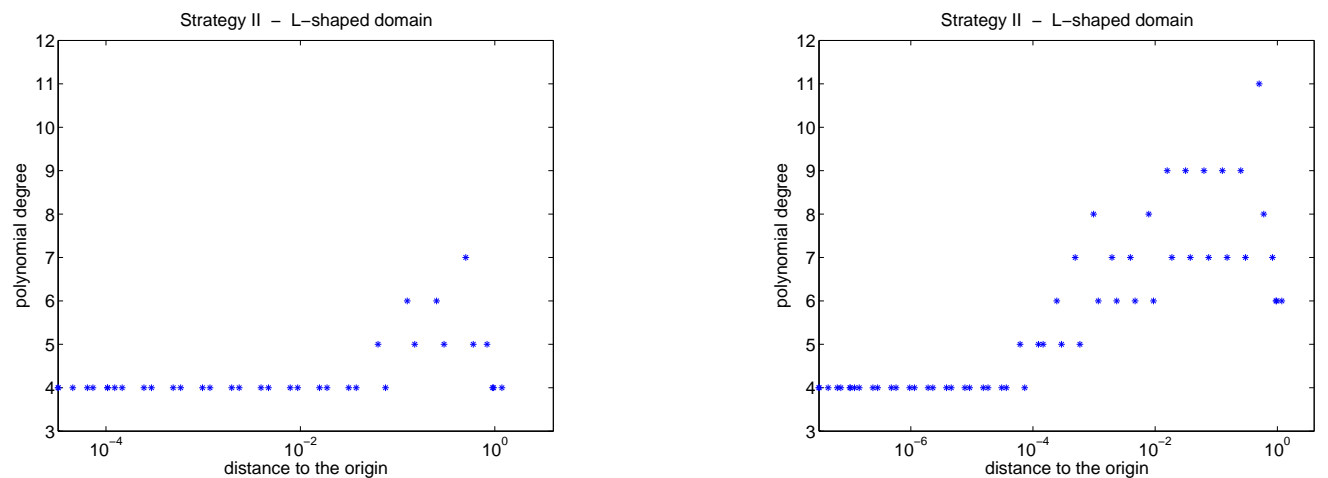

Figure 18: Strategy III - L-shaped domain - Polynomial degree distribution along the line from $(0,0)$ to $(-1 / 2,1)$ - Iteration levels 15 and 25

Figure 19: Comparison - Performance of $h p$-adaptive algorithm


Figure 20: Some properties of $\mathcal{E}_{\rho}:=\left\{z \in \mathbb{C}|| z+1|+| z-1 \mid \leq \rho+\rho^{-1}\right\}$

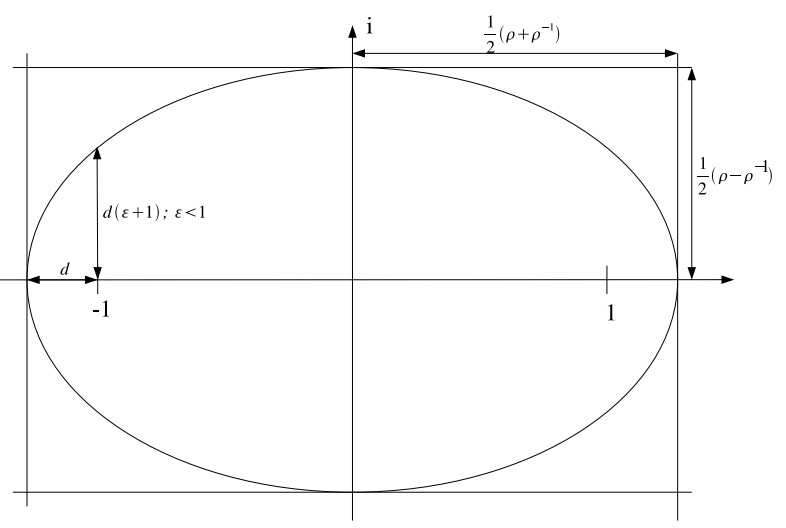




\section{Analytic functions on tetrahedra}

The $h p$-adaptive strategies of Section 4 can be applied to three-dimensional problems. In this section, we present the theoretical underpinning for the extension to 3D of Strategy II, which relies on estimating the decay of the coefficients when expanding a function in orthogonal polynomials. The main result of the present section is Theorem 5.7, which is the three-dimensional analog of Proposition 4.6.

We start with the introduction of some reference elements and transformations:

Definition 5.1 (reference elements). Let the reference triangle $\mathcal{T}^{2}$, the reference tetrahedron $\mathcal{T}^{3}$ and the $i$-dimensional reference cube $\mathcal{Q}^{i}$ be given by:

$$
\begin{aligned}
\mathcal{T}^{2} & =\{(x, y) \mid-1<x, y \wedge x+y<0\}, \\
\mathcal{T}^{3} & =\{(x, y, z) \mid-1<x, y, z \wedge x+y+z<-1\}, \\
\mathcal{Q}^{i} & =(-1,1)^{i} .
\end{aligned}
$$

Definition 5.2 (transformations). Let the transformations $D_{2}, D_{3}$ be given by:

$$
\begin{array}{ll}
D_{2} \quad: \quad\left(\eta_{1}, \eta_{2}\right) \mapsto\left(\frac{1}{2}\left(1+\eta_{1}\right)\left(1-\eta_{2}\right)-1, \eta_{2}\right) \\
D_{3} \quad: \quad\left(\eta_{1}, \eta_{2}, \eta_{3}\right) \mapsto\left(\frac{1}{4}\left(1+\eta_{1}\right)\left(1-\eta_{2}\right)\left(1-\eta_{3}\right)-1, \frac{1}{2}\left(1+\eta_{2}\right)\left(1-\eta_{3}\right)-1, \eta_{3}\right),
\end{array}
$$

Lemma 5.3 (inverse transformations). Let the transformations $D_{2}, D_{3}$ be given by Definition 5.2. Then the inverse maps are:

$$
\begin{aligned}
D_{2}^{-1} & =\left(\xi_{1}, \xi_{2}\right) \mapsto\left(2 \frac{1+\xi_{1}}{1-\xi_{2}}-1, \xi_{2}\right) \\
D_{3}^{-1} & =\left(\xi_{1}, \xi_{2}, \xi_{3}\right) \mapsto\left(2 \frac{1+\xi_{1}}{-\xi_{2}-\xi_{3}}-1,2 \frac{1+\xi_{2}}{1-\xi_{3}}-1, \xi_{3}\right)
\end{aligned}
$$

The following lemma shows an important relationship between the reference elements $\mathcal{T}^{i}$ and $\mathcal{Q}^{i}$.

Lemma 5.4. For $i=2,3$ let the reference elements $\mathcal{T}^{i}, \mathcal{Q}^{i}$ and the transformations $D_{i}$ be given by Definition 5.1, Definition 5.2. Then we have

$$
\mathcal{T}^{i}=D_{i}\left(\mathcal{Q}^{i}\right), \quad i=2,3
$$

Proof. Direct calculation. 


\subsection{Orthogonal polynomials on tetrahedra}

Similar to the case of the triangle in two dimensions, we introduce the set $\left(\psi_{p q r}\right)_{p, q, r \in \mathbb{N}_{0}}$ of polynomials that are orthogonal on the reference tetrahedron.

Definition 5.5 (orthogonal polynomials on the tetrahedron). Let $D_{3}$ be given by Definition 5.1 and denote by $P_{n}^{(\alpha, \beta)}(\eta)$ the $n$-th Jacobi polynomial with respect to the weight $(1-\eta)^{\alpha}(1+\eta)^{\beta}$. Then, for $p, q, r \in \mathbb{N}_{0}$, we define

$$
\psi_{p q r}=\tilde{\psi}_{p q r} \circ D_{3}^{-1}
$$

where $\tilde{\psi}_{p q r}(\eta)=\tilde{\psi}_{p q r}\left(\eta_{1}, \eta_{2}, \eta_{3}\right)$ is given by

$$
\tilde{\psi}_{p q r}(\eta)=P_{p}^{(0,0)}\left(\eta_{1}\right) P_{q}^{(2 p+1,0)}\left(\eta_{2}\right) P_{r}^{(2 p+2 q+2,0)}\left(\eta_{3}\right)\left(\frac{1-\eta_{2}}{2}\right)^{p}\left(\frac{1-\eta_{3}}{2}\right)^{p+q}
$$

The following lemma shows that the functions $\psi_{p q r}$ are $L^{2}$-orthogonal polynomials on the reference tetrahedron $\mathcal{T}^{3}$.

Lemma 5.6. Let the reference tetrahedron $T^{3}$ be given by Definition 5.1. Then the functions $\psi_{p q r}$ defined in Definition 5.5 satisfy $\psi_{p q r} \in \mathcal{P}_{p+q+r}\left(\mathcal{T}^{3}\right)$ and they are orthogonal on $\mathcal{T}^{3}$ with respect to the usual $L^{2}\left(\mathcal{T}^{3}\right)$ inner product. We have

$$
\left(\psi_{p q r}, \psi_{p^{\prime} q^{\prime} r^{\prime}}\right)_{L^{2}\left(\mathcal{T}^{3}\right)}=\frac{2}{(2 p+1)} \frac{2}{(2 p+2 q+2)} \frac{2}{(2 r+2 p+2 q+3)} \delta_{p^{\prime} p} \delta_{q^{\prime} q} \delta_{r^{\prime} r} .
$$

Proof. See [18] together with [20] where a two dimensional version of this Lemma is proved.

\subsection{Orthogonal polynomials and analytic functions}

The following theorem characterizes functions that analytic in a neighbourhood of a tetrahedron:

Theorem 5.7. Let the reference element $\mathcal{T}^{3}$ and the polynomials $\psi_{p q r}$ be given by Definition 5.1 and Definition 5.5. Let the function $u \in L^{2}\left(\mathcal{T}^{3}\right)$ be written as

$$
u=\sum_{p, q, r \in \mathbb{N}_{0}} u_{p q r} \psi_{p q r}
$$

Then $u$ is analytic on $\overline{\mathcal{T}}^{3}$ if and only if there exist constants $C, b>0$ such that

$$
\left|u_{p q r}\right| \leq C e^{-b(p+q+r)} \quad \forall p, q, r \in \mathbb{N}_{0} .
$$

Before proving this result, we need to introduce some notation: 
Definition 5.8. In the complex plane, for $\rho>1$, we introduce the ellipse $\mathcal{E}_{\rho}$ given by:

$$
\mathcal{E}_{\rho}:=\left\{z \in \mathbb{C}|| z+1|+| z-1 \mid \leq \rho+\rho^{-1}\right\} .
$$

Remark 5.9. A calculation shows that

$$
\operatorname{dist}\left(\partial \mathcal{E}_{\rho}, 1\right)=\frac{(\rho-1)^{2}}{2 \rho} .
$$

Lemma 5.10. For $\alpha, \beta>-1$ denote by $P_{q}^{(\alpha, \beta)}(\eta)$ the $q$-th Jacobi polynomial with respect to the weight $(1-\eta)^{\alpha}(1+\eta)^{\beta}$. Then, for each $q \in \mathbb{N}_{0}$, the function

$$
w \mapsto \tilde{Q}_{q}^{(\alpha, \beta)}(w)=\int_{-1}^{1}(1-t)^{\alpha}(1+t)^{\beta} \frac{P_{q}^{(\alpha, \beta)}(t)}{w-t} d t
$$

is holomorphic on $\mathbb{C} \backslash[-1,1]$ and for $\rho>1$ we have

$$
\begin{aligned}
\left|\tilde{Q}_{q}^{(0,0)}(w)\right| & \leq \frac{2 \pi}{1-1 / \rho} \rho^{-(q+1)} \quad \forall w \in \partial \mathcal{E}_{\rho}, \\
\left|\tilde{Q}_{q}^{(\alpha, 0)}(w)\right| & \leq \frac{2^{\alpha+2}}{\alpha+1} \frac{q+2}{(1-1 / \rho)^{2}} \rho^{-(q+1)} \quad \forall w \in \partial \mathcal{E}_{\rho} .
\end{aligned}
$$

Proof. [20, Lemma 3.2.10, Corollary 3.2.11]

Proof of Theorem 5.7. First, we assume (8) and show that $\sum_{p, q, r \in \mathbb{N}_{0}} u_{p q r} \psi_{p q r}$ represents an analytic function. To that end, we chose $\rho>1$ so small that $\ln \rho \leq b / 2$ and set

$$
u_{k}:=\sum_{p+q+r \leq k} u_{p q r} \psi_{p q r}, \quad k=0,1, \ldots,
$$

Lemma 5.14 ensures the existence of an open complex neighborhood $\mathcal{T}^{\prime}$ of $\overline{\mathcal{T}}^{3}$ such that

$$
\left\|\psi_{p q r}\right\|_{L^{\infty}\left(\mathcal{T}^{\prime}\right)} \leq e^{(b / 2)(p+q+r)} .
$$

In combination with $(8)$ we obtain that the sequence $\left(u_{k}\right)_{k=0}^{\infty}$ converges uniformly on $\mathcal{T}^{\prime}$. Since all the functions $u_{k}$ are analytic on $\mathcal{T}^{\prime}$, the limit function $u$ is analytic on $\mathcal{T}^{\prime}$ (see, e.g., [16, Cor. 2.2.4]).

We now turn to the second part of the theorem, where we show that analyticity of $u$ on a neighborhood of the closure of the reference tetrahedron $\mathcal{T}^{3}$. Since the polynomials $\psi_{p q r}$ are $L^{2}\left(\mathcal{T}^{3}\right)$-orthogonal, we compute

$$
u_{p q r}=\frac{\left(\psi_{p q r}, u\right)_{L^{2}\left(\mathcal{T}^{3}\right)}}{\left\|\psi_{p q r}\right\|_{L^{2}\left(\mathcal{T}^{3}\right)}^{2}}=\frac{1}{\left\|\psi_{p q r}\right\|_{L^{2}\left(\mathcal{T}^{3}\right)}^{2}} \int_{\mathcal{T}^{3}} u \psi_{p q r} \mathrm{~d} \Omega .
$$

We need to show the existence of $C, b>0$ such that

$$
\left|\left(\psi_{p q r}, u\right)_{L^{2}\left(\mathcal{T}^{3}\right)}\right| \leq C e^{-b(p+q+r)} .
$$


Denoting by $U_{p q}$ the function defined in Lemma 5.13, the transformation of $\mathcal{T}^{3}$ to the cube $\mathcal{Q}^{3}$ via $D_{3}$ yields

$$
\left|\left(\psi_{p q r}, u\right)_{L^{2}\left(\mathcal{T}^{3}\right)}\right|=\left|\int_{-1}^{1} P_{r}^{(2 p+2 q+2,0)}\left(\eta_{3}\right)\left(\frac{1-\eta_{3}}{2}\right)^{(p+q+2)} U_{p q}\left(\eta_{3}\right) \mathrm{d} \eta_{3}\right| .
$$

Lemma 5.13 asserts that $U_{p q}$ is holomorphic on $\mathcal{E}_{\rho}$ for some $\rho>1$ and has a zero of multiplicity $p+q$ at $\eta_{3}=1$. Hence, we may apply Cauchy's integral theorem to the holomorphic function $\eta_{3} \mapsto U_{p q}\left(\eta_{3}\right) /\left(1-\eta_{3}\right)^{(p+q)}$ and obtain a first bound for $\left(\psi_{p q r}, u\right)_{L^{2}\left(\mathcal{T}^{3}\right)}$ :

$$
\begin{aligned}
\left|\left(\psi_{p q r}, u\right)_{L^{2}\left(\mathcal{T}^{3}\right)}\right| & \left|2^{-p-q-2} \int_{-1}^{1}\left(1-\eta_{3}\right)^{(2 p+2 q+2)} \frac{U_{p q}\left(\eta_{3}\right)}{\left(1-\eta_{3}\right)^{p+q}} P_{r}^{(2 p+2 q+2,0)}\left(\eta_{3}\right) \mathrm{d} \eta_{3}\right| \\
& =\left|\frac{2^{-(p+q+2)}}{2 \pi \mathbf{i}} \oint_{\zeta_{3} \in \partial \mathcal{E}_{\rho}} \frac{U_{p q}\left(\zeta_{3}\right)}{\left(1-\zeta_{3}\right)^{p+q}} \tilde{Q}_{r}^{(2 p+2 q+2,0)}\left(\zeta_{3}\right) \mathrm{d} \zeta_{3}\right| \\
& \leq C 2^{-(p+q+2)} \frac{\operatorname{length}\left(\partial \mathcal{E}_{\rho}\right)}{\left(\operatorname{dist}\left(\partial \mathcal{E}_{\rho}, 1\right)\right)^{(p+q)}}\left\|U_{p q}\right\|_{L^{\infty}\left(\partial \mathcal{E}_{\rho}\right)}\left\|\tilde{Q}_{r}^{(2 p+2 q+2,0)}\right\|_{L^{\infty}\left(\partial \mathcal{E}_{\rho}\right)} \\
& \leq C 2^{-(p+q+2)} \rho\left(\frac{2 \rho}{(\rho-1)^{2}}\right)^{p+q} e^{-b(p+q)} \frac{2^{(2 p+2 q+2)}}{(2 p+2 q+3)} \frac{(r+2)}{(1-1 / \rho)^{2}} \rho^{-(r+1)} \\
& \leq C \gamma^{p+q} \delta^{-r},
\end{aligned}
$$

with $\delta>1$ and $C, \gamma, \delta$ independent of $p, q, r$. Next, making use of the Cauchy-Schwarz inequality, exploiting orthogonality relations of Jacobi polynomials and Lemma 5.13 we obtain a second bound for $\left(\psi_{p q r}, u\right)_{L^{2}\left(\mathcal{T}^{3}\right)}$

$$
\begin{aligned}
\left|\left(\psi_{p q r}, u\right)_{L^{2}\left(\mathcal{T}^{3}\right)}\right|= & \left|\int_{-1}^{1} P_{r}^{(2 p+2 q+2,0)}\left(\eta_{3}\right)\left(\frac{1-\eta_{3}}{2}\right)^{(p+q+2)} U_{p q}\left(\eta_{3}\right) \mathrm{d} \eta_{3}\right| \\
\leq & \left(\int_{-1}^{1}\left(\frac{1-\eta_{3}}{2}\right)^{(2 p+2 q+2)}\left(P_{r}^{(2 p+2 q+2,0)}\left(\eta_{3}\right)\right)^{2} \mathrm{~d} \eta_{3}\right)^{\frac{1}{2}} \times \\
& \left(\int_{-1}^{1}\left(\frac{1-\eta_{3}}{2}\right)^{2}\left|U_{p q}\left(\eta_{3}\right)\right|^{2} \mathrm{~d} \eta_{3}\right)^{\frac{1}{2}} \\
\leq & C\left(\frac{2}{2 p+2 q+2 r+3}\right)^{\frac{1}{2}} e^{-b(p+q)} \leq C e^{-b(p+q)} .
\end{aligned}
$$

We combine inequality (11) and (12) to achieve

$$
\left|u_{p q r}\right| \leq C \min \left\{e^{-b(p+q)}, \gamma^{p+q} \delta^{-r}\right\} .
$$

If $\gamma<1$, then (13) implies immediately (9). We may therefore assume $\gamma \geq 1$. We choose $\lambda>0$ such that $\gamma^{\lambda} / \delta=: q<1$ and distinguish two cases: 
1. For $(p+q) \leq \lambda r$ we have

$$
\left|u_{p q r}\right| \leq C \gamma^{p+q} \delta^{-r} \leq C\left(\gamma^{\lambda} / \delta\right)^{r}=C q^{r}=C q^{r / 2+r / 2} \leq C q^{\frac{1}{2} \min \{1,1 / \lambda\}(r+p+q)},
$$

which is the desired bound (9).

2. For $(p+q)>\lambda r$ we have

$$
(p+q) \geq \frac{\lambda}{2}(p+q+r)
$$

and the desired result follows from

$$
\left|u_{p q r}\right| \leq C e^{-b(p+q)} \leq C e^{-b \frac{\lambda}{2}(p+q+r)} \leq C e^{-b^{\prime}(p+q+r)} .
$$

\subsection{Auxiliary Results}

Lemma 5.11. Let $D_{3}$ be given by Definition 5.2 and let $u$ be analytic on $\overline{\mathcal{T}}^{3}$. Then there exist $C>0, \delta>0, \rho>1$ depending only on $u$ such that:

1. The function $u \circ D_{3}$ is holomorphic on $\overline{\mathcal{Q}}^{3}$ and can be extended to a function $\tilde{u}$ holomorphic on $\mathcal{E}_{\rho} \times \mathcal{E}_{\rho} \times \mathcal{E}_{\rho}$ with

$$
\|\tilde{u}\|_{L^{\infty}\left(\mathcal{E}_{\rho}^{3}\right)} \leq C .
$$

2. For all $\eta_{2}, \eta_{3} \in(-1,1)$ the function $\eta_{1} \mapsto \tilde{u}\left(\eta_{1}, \eta_{2}, \eta_{3}\right)$ is holomorphic on $\mathcal{E}_{1+\delta /\left(\left(1-\eta_{2}\right)\left(1-\eta_{3}\right)\right)}$ and we have

$$
\sup _{\left(\eta_{2}, \eta_{3}\right) \in \mathcal{Q}^{2}}\left\|\tilde{u}\left(\cdot, \eta_{2}, \eta_{3}\right)\right\|_{L^{\infty}\left(\mathcal{E}_{1+\delta /\left(\left(1-\eta_{2}\right)\left(1-\eta_{3}\right)\right)}\right)} \leq C .
$$

3. For all $\eta_{1}, \eta_{3} \in(-1,1)$ the function $\eta_{2} \mapsto \tilde{u}\left(\eta_{1}, \eta_{2}, \eta_{3}\right)$ is holomorphic on $\mathcal{E}_{1+\delta /\left(1-\eta_{3}\right)}$ and we have

$$
\sup _{\left(\eta_{1}, \eta_{3}\right) \in \mathcal{Q}^{2}}\left\|\tilde{u}\left(\eta_{1}, \cdot, \eta_{3}\right)\right\|_{L^{\infty}\left(\mathcal{E}_{1+\delta /\left(1-\eta_{3}\right)}\right)} \leq C .
$$

Proof. Since $u$ is analytic on $\overline{\mathcal{T}}^{3}$, there exists a complex open neighbourhood $\mathcal{T}^{\prime} \subset \mathbb{C}^{3}$ of $\bar{T}^{3}$ such that $u$ is holomorphic and bounded on $\mathcal{T}^{\prime}$. Thus, because of the continuity of $D_{3}$, there exists $\rho>1$ such that $D_{3}\left(\mathcal{E}_{\rho}^{3}\right) \subset \mathcal{T}^{\prime}$ and the first claim is proved. In order to prove the second claim, we have to show that for arbitrary $\epsilon>0$ there exists $\delta(\epsilon)>0$ such that

$$
\left(\eta_{1}, \eta_{2}, \eta_{3}\right) \in G_{\delta}:=\left\{\left(\eta_{1}, \eta_{2}, \eta_{3}\right) \mid \eta_{1} \in \mathcal{E}_{\rho_{1}},\left(\eta_{2}, \eta_{3}\right) \in \mathcal{Q}^{2}\right\}
$$

with

$$
\rho_{1}=1+\frac{\delta}{\left(1-\eta_{2}\right)\left(1-\eta_{3}\right)}
$$

implies

$$
\inf _{\mathbf{x} \in \bar{T}^{3}}\left|D_{3}\left(\eta_{1}, \eta_{2}, \eta_{3}\right)-\mathbf{x}\right| \leq \epsilon .
$$

To that end, we set $\eta_{1}=a+b \mathbf{i}, \delta<\frac{8}{\sqrt{5}} \epsilon$ and distinguish three cases: 
1. For $a \leq-1$ we have $A_{1}:=D_{3}\left(-1, \eta_{2}, \eta_{3}\right) \in \overline{\mathcal{T}}^{3}$ with:

$$
\begin{aligned}
& \left|D_{3}\left(\eta_{1}, \eta_{2}, \eta_{3}\right)-A_{1}\right| \\
& \quad=\frac{1}{4}\left(1-\eta_{2}\right)\left(1-\eta_{3}\right)\left|\eta_{1}+1\right| \leq \frac{\sqrt{5}}{4}\left(1-\eta_{2}\right)\left(1-\eta_{3}\right)\left|\frac{1}{2}\left(\rho_{1}+\rho_{1}^{-1}\right)-1\right| \\
& \quad=\frac{1}{4}\left(1-\eta_{2}\right)\left(1-\eta_{3}\right) \frac{\sqrt{5}}{2} \frac{\delta}{\left(1-\eta_{2}\right)\left(1-\eta_{3}\right)} \cdot \frac{\delta}{\left(1-\eta_{2}\right)\left(1-\eta_{3}\right)+\delta}<\epsilon .
\end{aligned}
$$

2. For $|a|<1$ we have $A_{2}:=D_{3}\left(a, \eta_{2}, \eta_{3}\right) \in \overline{\mathcal{T}}^{3}$ with:

$$
\begin{aligned}
& \left|D_{3}\left(\eta_{1}, \eta_{2}, \eta_{3}\right)-A_{2}\right| \\
& \quad=\frac{1}{4}\left(1-\eta_{2}\right)\left(1-\eta_{3}\right)|b| \leq \frac{1}{8}\left(1-\eta_{2}\right)\left(1-\eta_{3}\right)\left|\rho_{1}-\rho_{1}^{-1}\right| \\
& \quad=\frac{1}{8}\left(1-\eta_{2}\right)\left(1-\eta_{3}\right)\left|\frac{\delta}{\left(1-\eta_{2}\right)\left(1-\eta_{3}\right)} \cdot \frac{2\left(1-\eta_{2}\right)\left(1-\eta_{3}\right)+\delta}{\left(1-\eta_{2}\right)\left(1-\eta_{3}\right)+\delta}\right|<\epsilon .
\end{aligned}
$$

3. For $a \geq 1$ we have $A_{3}:=D_{3}\left(1, \eta_{2}, \eta_{3}\right) \in \overline{\mathcal{T}}^{3}$ and obtain analogously to the case $a \leq-1$ :

$$
\left|D_{3}\left(\eta_{1}, \eta_{2}, \eta_{3}\right)-A_{3}\right| \leq \frac{\sqrt{5}}{8} \delta<\epsilon
$$

To prove the third claim we proceed similarly.

Lemma 5.12. Let the transformation $D_{3}$ be given by Definition 5.2 and let the function $u$ be analytic on $\overline{\mathcal{T}}^{3}$. For $p \in \mathbb{N}_{0}$ let the function $\left(\eta_{2}, \eta_{3}\right) \mapsto U_{p}\left(\eta_{2}, \eta_{3}\right)$ be defined by:

$$
U_{p}\left(\eta_{2}, \eta_{3}\right):=\int_{-1}^{1} P_{p}^{(0,0)}\left(\eta_{1}\right)\left[u \circ D_{3}\right]\left(\eta_{1}, \eta_{2}, \eta_{3}\right) d \eta_{1}
$$

Then there exist $\rho>1, \delta>0, C>0$ depending only on $u$ such that:

1. The function $U_{p}$ is holomorphic and bounded on $\mathcal{E}_{\rho} \times \mathcal{E}_{\rho}$ with

$$
\left\|U_{p}\right\|_{L^{\infty}\left(\mathcal{E}_{\rho} \times \mathcal{E}_{\rho}\right)} \leq C \rho^{-p}
$$

2. For each $\eta_{3} \in(-1,1)$ the function $\eta_{2} \mapsto U_{p}\left(\eta_{2}, \eta_{3}\right)$ is holomorphic on $\mathcal{E}_{1+\delta /\left(1-\eta_{3}\right)}$ with

$$
\sup _{\eta_{3} \in(-1,1)}\left\|U_{p}\left(\cdot, \eta_{3}\right)\right\|_{L^{\infty}\left(\mathcal{E}_{1+\delta /\left(1-\eta_{3}\right)}\right)} \leq C .
$$

3. The function $U_{p}$ has zeros of multiplicity $p$ at $\eta_{2}=1$ and $\eta_{3}=1$. 
Proof. The second claim follows from Lemma 5.11-3 and $U_{p}$ holomorphic on $\mathcal{E}_{\rho} \times \mathcal{E}_{\rho}$ follows from Lemma 5.11-1. To prove $\left\|U_{p}\right\|_{L^{\infty}\left(\mathcal{E}_{\rho} \times \mathcal{E}_{\rho}\right)} \leq C \rho^{-p}$, we exploit Cauchy's integral representation formula and obtain with Lemma 5.10:

$$
\begin{aligned}
\left|U_{p}\left(\zeta_{2}, \zeta_{3}\right)\right| & =\left|\frac{1}{2 \pi \mathbf{i}} \int_{-1}^{1} \oint_{\zeta_{1} \in \partial \mathcal{E}_{\rho}} \frac{\tilde{u}\left(\zeta_{1}, \zeta_{2}, \zeta_{3}\right)}{\zeta_{1}-\eta_{1}} P_{p}^{(0,0)}\left(\eta_{1}\right) \mathrm{d} \zeta_{1} \mathrm{~d} \eta_{1}\right| \\
& =\left|\frac{1}{2 \pi \mathbf{i}} \oint_{\zeta_{1} \in \partial \mathcal{E}_{\rho}} \tilde{u}\left(\zeta_{1}, \zeta_{2}, \zeta_{3}\right) \tilde{Q}_{p}^{(0,0)}\left(\zeta_{1}\right) \mathrm{d} \zeta_{1}\right| \\
& \leq \frac{\operatorname{length}\left(\mathcal{E}_{\rho}\right)}{2 \pi}\|\tilde{u}\|_{L^{\infty}\left(\mathcal{E}_{\rho}^{3}\right)}\left\|\tilde{Q}_{p}^{(0,0)}\right\|_{L^{\infty}\left(\partial \mathcal{E}_{\rho}\right)} \leq C \rho^{-p},
\end{aligned}
$$

By Lemma 5.12-1 $U_{p}$ is holomorphic on $\mathcal{E}_{\rho} \times \mathcal{E}_{\rho}$. In order to show that it has a zero of multiplicity $p$ at $\eta_{2}=1$ and $\eta_{3}=1$, it suffices to prove the existence of $C>0$ independent of $\eta_{2}, \eta_{3}$ such that

$$
\left|U_{p}\left(\eta_{2}, \eta_{3}\right)\right| \leq C\left(1-\eta_{2}\right)^{p}\left(1-\eta_{3}\right)^{p} \quad \forall \eta_{2}, \eta_{3} \in(-1,1) .
$$

Lemma 5.11-2 together with Cauchy's integral representation formula yields

$$
\left|U_{p}\left(\eta_{2}, \eta_{3}\right)\right|=\left|\frac{1}{2 \pi \mathbf{i}} \int_{-1}^{1} \oint_{\zeta_{1} \in \partial \mathcal{E}_{\rho_{1}}} \frac{\tilde{u}\left(\zeta_{1}, \eta_{2}, \eta_{3}\right)}{\zeta_{1}-\eta_{1}} P_{p}^{(0,0)}\left(\eta_{1}\right) \mathrm{d} \zeta_{1} \mathrm{~d} \eta_{1}\right|
$$

with $\rho_{1}$ given by (14). Appealing again to Lemma 5.10, we get

$$
\begin{aligned}
\left|U_{p}\left(\eta_{2}, \eta_{3}\right)\right| & \leq \frac{\operatorname{length}\left(\partial \mathcal{E}_{\rho_{1}}\right)}{2 \pi}\|\tilde{u}\|_{L^{\infty}\left(G_{\delta}\right)}\left\|\tilde{Q}_{p}^{(0,0)}\right\|_{L^{\infty}\left(\partial \mathcal{E}_{\rho_{1}}\right)} \\
& \leq C\left(\frac{\left(1-\eta_{2}\right)\left(1-\eta_{3}\right)}{\delta+\left(1-\eta_{2}\right)\left(1-\eta_{3}\right)}\right)^{p}
\end{aligned}
$$

where $C$ depends solely on $u$. Since $\delta>0$ and $\eta_{2}, \eta_{3} \in(-1,1)$, we arrive at

$$
\left|U_{p}\left(\eta_{2}, \eta_{3}\right)\right| \leq C \delta^{-p}\left(1-\eta_{2}\right)^{p}\left(1-\eta_{3}\right)^{p}
$$

which is the desired bound.

Lemma 5.13. Let the transformation $D_{3}$ be given by Definition 5.2 and let the function $u$ be analytic on $\overline{\mathcal{T}}^{3}$. For $p, q \in \mathbb{N}_{0}$ let the function $\eta_{3} \mapsto U_{p q}\left(\eta_{3}\right)$ be defined by:

$$
U_{p q}\left(\eta_{3}\right)=\int_{-1}^{1} U_{p}\left(\eta_{2}, \eta_{3}\right) P_{q}^{(2 p+1,0)}\left(\eta_{2}\right)\left(\frac{1-\eta_{2}}{2}\right)^{p+1} d \eta_{2},
$$

where $U_{p}$ denotes the function of Lemma 5.12. Then there exist $\rho>1, b>0, C>0$ depending only on u such that: 
1. The function $U_{p q}$ is holomorphic on $\mathcal{E}_{\rho}$ and has a zero of multiplicity $(p+q)$ at $\eta_{3}=1$.

2. For all $\zeta_{3} \in \mathcal{E}_{\rho}$

$$
\left|U_{p q}\left(\zeta_{3}\right)\right| \leq C e^{-b(p+q)} .
$$

Proof. The holomorphy of $U_{p q}$ follow from Lemma 5.12-1. To show that $U_{p q}$ has a zero of multiplicity $(p+q)$ at $\eta_{3}=1$, it suffices to show the existence of $C$ independent of $\eta_{3}$ such that

$$
\left|U_{p q}\left(\eta_{3}\right)\right| \leq C\left(1-\eta_{3}\right)^{p+q} \quad \forall \eta_{3} \in(-1,1) .
$$

From Lemma 5.12 we know that for each $\eta_{3} \in(-1,1)$ the function $U_{p}\left(\cdot, \eta_{3}\right)$ is holomorphic on $\mathcal{E}_{\rho_{2}}=\mathcal{E}_{1+\delta /\left(1-\eta_{3}\right)}$ and has a zero of multiplicity $p$ at $\eta_{2}=1$. Hence, we may apply Cauchy's integral theorem to the holomorphic function $\eta_{2} \mapsto U_{p}\left(\eta_{2}, \eta_{3}\right) /\left(1-\eta_{2}\right)^{p}$ to arrive at

$$
\begin{aligned}
U_{p q}\left(\eta_{3}\right) & =\frac{1}{2 \pi \mathbf{i}}\left(\frac{1}{2}\right)_{\zeta_{2} \in \partial \mathcal{E}_{\rho_{2}}}^{p+1} \frac{U_{p}\left(\zeta_{2}, \eta_{3}\right)}{\left(1-\zeta_{2}\right)^{p}} \int_{-1}^{1} \frac{P_{q}^{(2 p+1,0)}\left(\eta_{2}\right)}{\zeta_{2}-\eta_{2}}\left(1-\eta_{2}\right)^{2 p+1} \mathrm{~d} \eta_{2} \mathrm{~d} \zeta_{2} \\
& =C \oint_{\zeta_{2} \in \partial \mathcal{E}_{\rho_{2}}} \frac{U_{p}\left(\zeta_{2}, \eta_{3}\right)}{\left(1-\zeta_{2}\right)^{p}} \tilde{Q}_{q}^{(2 p+1,0)}\left(\zeta_{2}\right) \mathrm{d} \zeta_{2} .
\end{aligned}
$$

Thus, we obtain

$$
\begin{aligned}
\left|U_{p q}\left(\eta_{3}\right)\right| & \leq C \frac{\operatorname{length}\left(\partial \mathcal{E}_{\rho_{2}}\right)}{\left(\operatorname{dist}\left(\partial \mathcal{E}_{\rho_{2}}, 1\right)\right)^{p}}\left\|\tilde{Q}_{q}^{(2 p+1,0)}\right\|_{L^{\infty}\left(\partial \mathcal{E}_{\rho_{2}}\right)}\left\|U_{p}\left(\cdot, \eta_{3}\right)\right\|_{L^{\infty}\left(\mathcal{E}_{\rho_{2}}\right)} \\
& \leq C \rho_{2}\left(\frac{\left(\rho_{2}-1\right)^{2}}{2 \rho_{2}}\right)^{-p}\left(\frac{2^{2 p+3}}{2 p+2} \frac{q+2}{\left(1-1 / \rho_{2}\right)^{2}} \rho_{2}^{-(q+1)}\right) \\
& \leq C \frac{\rho_{2}^{p-q+2}}{\left(\rho_{2}-1\right)^{2 p+2}} \leq C \frac{\left(1-\eta_{3}+\delta\right)^{p-q+2}}{\delta^{2 p+2}}\left(1-\eta_{3}\right)^{p+q}
\end{aligned}
$$

where $C$ is independent of $\eta_{3}$. Since $\delta>0$ and $\eta_{3} \in(-1,1)$, we arrive at

$$
\left|U_{p q}\left(\eta_{3}\right)\right| \leq C\left(1-\eta_{3}\right)^{p+q}
$$

which is the desired bound. We proceed similarly for the second claim. We use the bound (16) but take as the contour of integration $\partial \mathcal{E}_{\rho}$, i.e.,

$$
\begin{aligned}
\left|U_{p q}\left(\zeta_{3}\right)\right| & =C\left|\oint_{\zeta_{2} \in \partial \mathcal{E}_{\rho}} \frac{U_{p}\left(\zeta_{2}, \zeta_{3}\right)}{\left(1-\zeta_{2}\right)^{p}} \tilde{Q}_{q}^{(2 p+1,0)}\left(\zeta_{2}\right) \mathrm{d} \zeta_{2}\right| \\
& \leq C \frac{\operatorname{length}\left(\partial \mathcal{E}_{\rho}\right)}{\left(\operatorname{dist}\left(\partial \mathcal{E}_{\rho}, 1\right)\right)^{p}}\left\|\tilde{Q}_{q}^{(2 p+1,0)}\right\|_{L^{\infty}\left(\partial \mathcal{E}_{\rho}\right)}\left\|U_{p}\left(\zeta_{2}, \zeta_{3}\right)\right\|_{L^{\infty}\left(\mathcal{E}_{\rho}^{2}\right)} \\
& \leq C \rho\left(\frac{2 \rho}{(\rho-1)^{2}}\right)^{p} \frac{2^{2 p+3}}{2 p+2} \frac{q+2}{(1-1 / \rho)^{2}} \rho^{-(q+1)} \leq C \rho^{-q} \gamma^{p}
\end{aligned}
$$


with $C, \gamma$ independent of $p, q$, and $\eta_{3}$. A second bound for $\left|U_{p q}\left(\zeta_{3}\right)\right|$ follows from the Cauchy-Schwarz inequality together with Lemma 5.12-1 and basic properties of Jacobi polynomials:

$$
\begin{aligned}
\left|U_{p q}\left(\zeta_{3}\right)\right| & =\int_{-1}^{1} U_{p}\left(\eta_{2}, \zeta_{3}\right) P_{q}^{(2 p+1,0)}\left(\eta_{2}\right)\left(\frac{1-\eta_{2}}{2}\right)^{p+1} \mathrm{~d} \eta_{2} \\
& \leq\left\{\int_{-1}^{1}\left|U_{p}\left(\eta_{2}, \zeta_{3}\right)\right|^{2}\left(\frac{1-\eta_{2}}{2}\right) \mathrm{d} \eta_{2}\right\}^{\frac{1}{2}}\left\{\int_{-1}^{1}\left(P_{q}^{(2 p+1,0)}\left(\eta_{2}\right)\right)^{2}\left(\frac{1-\eta_{2}}{2}\right)^{2 p+1} \mathrm{~d} \eta_{2}\right\}^{\frac{1}{2}} \\
& \leq C \rho^{-p} \frac{2}{2 p+2 q+2} \leq C \rho^{-p} .
\end{aligned}
$$

Combining the last two bounds as in Theorem 5.7 gives the desired result

$$
\left|U_{p q}\left(\zeta_{3}\right)\right| \leq C e^{-b(p+q)} \quad \forall \zeta_{3} \in \mathcal{E}_{\rho}
$$

Lemma 5.14. Let $\psi_{p q r}$ be given by Definition 5.5. Then for arbitrary $\rho>1$ there exists $C$ and an open complex neighborhood $\mathcal{T}^{\prime} \subset \mathbb{C}^{3}$ with $\overline{\mathcal{T}}^{3} \subset \mathcal{T}^{\prime}$ such that

$$
\left\|\psi_{p q r}\right\|_{L^{\infty}\left(\mathcal{T}^{\prime}\right)} \leq C(p+q+r)^{3} \rho^{p+q+r} \quad \forall p, q, r \in \mathbb{N}_{0} .
$$

Proof. For univariate polynomials we have (see [10, Chap 4, Thm. 2.2])

$$
\|u\|_{L^{\infty}\left(\mathcal{E}_{\rho}\right)} \leq \rho^{p}\|u\|_{L^{\infty}(-1,1)} \quad \forall \rho>1 \quad \forall u \in \mathcal{P}_{p}
$$

Thus, by tensor product arguments, we obtain

$$
\|u\|_{L^{\infty}\left(\mathcal{E}_{\rho^{1 / 3}}^{3}\right)} \leq \rho^{p}\|u\|_{L^{\infty}\left(\mathcal{Q}^{3}\right)} \quad \forall \rho>1 \quad \forall u \in Q_{p}\left(\mathcal{Q}^{3}\right),
$$

which implies that for arbitrary $\rho>1$ there exists an open complex neighborhood $\mathcal{Q}^{\prime} \supset \overline{\mathcal{Q}}^{3}$ such that

$$
\|u\|_{L^{\infty}\left(\mathcal{Q}^{\prime}\right)} \leq \rho^{p}\|u\|_{L^{\infty}\left(\overline{\mathcal{Q}}^{3}\right)} \quad \forall u \in Q_{p}\left(\mathcal{Q}^{3}\right) .
$$

An affine change of variables shows that (17) holds for an arbitrary closed parallelepiped $P$. That is, for all $\rho>1$ there exists an open complex neighborhood $P^{\prime}$ of $P$ such that

$$
\|u\|_{L^{\infty}\left(\mathcal{P}^{\prime}\right)} \leq \rho^{p}\|u\|_{L^{\infty}(\overline{\mathcal{P}})} \quad \forall u \in Q_{p}\left(\mathcal{Q}^{3}\right)
$$

Now, since it is possible to find, for example, ten not necessarily disjoint parallelepipeds $P^{1}, \ldots, P^{10}$ such that

$$
\overline{\mathcal{T}}^{3}=\bigcup_{i=1}^{10} \bar{P}^{i}
$$


we obtain for each $\rho>1$ a complex neighborhood $\mathcal{T}^{\prime}:=\cup_{i=1}^{10} P^{\prime i}$ of $\overline{\mathcal{T}}^{3}$ with $P^{\prime i}$ given by (18) and

$$
\|u\|_{L^{\infty}\left(\mathcal{T}^{\prime}\right)}=\max _{i=1}^{10}\|u\|_{L^{\infty}\left(P^{\prime i}\right)} \leq \rho^{p} \max _{i=1}^{10}\|u\|_{L^{\infty}\left(\bar{P}^{i}\right)} \leq \rho^{p}\|u\|_{L^{\infty}\left(\overline{\mathcal{T}}^{3}\right)}
$$

for all $u \in \mathcal{P}_{p}\left(\mathcal{T}^{3}\right)$. In order to replace the $L^{\infty}$ bound on the right-hand side by an $L^{2}$-bound, we need the following polynomial inverse estimate:

$$
\|u\|_{L^{\infty}\left(\overline{\mathcal{T}}^{3}\right)} \leq C p^{3}\|u\|_{L^{2}\left(\overline{\mathcal{T}}^{3}\right)} \quad \forall u \in \mathcal{P}_{p}\left(\mathcal{T}^{3}\right)
$$

this estimate can be obtained using the same arguments as in the two-dimensional situation proved in [25, Thm. 4.76]. Inserting (20) into (19) gives for the polynomial $\psi_{p q r}$

$$
\left\|\psi_{p q r}\right\|_{L^{\infty}\left(\mathcal{T}^{\prime}\right)} \leq C(p+q+r)^{3} \rho^{p+q+r}\left\|\psi_{p q r}\right\|_{L^{2}\left(\mathcal{T}^{3}\right)} .
$$

The claim of the lemma now follows from Lemma 5.6, which gives us the bound $\left\|\psi_{p q r}\right\|_{L^{2}\left(\mathcal{T}^{3}\right)} \leq 2 / \sqrt{3}$ for all $p, q, r \in \mathbb{N}_{0}$.

\section{References}

[1] M. Ainsworth and B. Senior. Aspects of an adaptive $h p$-finite element method: adaptive strategy, conforming approximation and efficient solvers. Comput. Meth. Appl. Mech. Engrg., 150:65-87, 1997.

[2] M. Ainsworth and B. Senior. An adaptive refinement strategy for $h p$-finite-element computations. Appl. Numer. Math., 26:165-178, 1998.

[3] M. Ainsworth and B. Senior. $h p$-finite element procedures on non-uniform geometric meshes. In M. Bern, J. Flaherty, and M. Luskin, editors, Grid generation and adaptive algorithms, pages 1-27. Springer Verlag, 1999. IMA Vol. Math. Appl. 113.

[4] I. Babuška and B.Q. Guo. The $h-p$ version of the finite element method. Part 1: The basic approximation results. Computational Mechanics, 1:21-41, 1986.

[5] I. Babuška and M. Suri. The optimal convergence rate of the $p$-version of the finite element method. SIAM J. Numer. Anal., 24:750-776, 1987.

[6] C. Bernardi, R.G. Owens, and J. Valenciano. An error indicator for mortar element solutions to the Stokes problem. IMA J. Numer. Anal., 21:857-886, 2001.

[7] E. Bertóti and B. Szabó. adaptive selection of polynomial degrees on a finite element mesh. Internat. J. Numer. Meths. Engrg., 42:561-578, 1998.

[8] L. Demkowicz, W. Rachowicz, and Ph. Devloo. A fully automatic hp-adaptivity. J. Sci. Comput., 17:117-142, 2002. 
[9] L. Demkowicz and P. Solin. Goal oriented hp-adaptivity for elliptic problems. Comput. Meth. Appl. Mech. Engrg., 193:449-468, 2004.

[10] R.A. DeVore and G.G. Lorentz. Constructive Approximation. Springer Verlag, 1993.

[11] T. Eibner. Algorithmik der randkonzentrierten FEM. PhD thesis, TU Chemnitz, (in prep.).

[12] T. Eibner and J.M. Melenk. A fast and efficient method for setting up the stiffness matrix in hp-fem. (in prep.).

[13] H. Goering, H.G. Roos, and L. Tobiska. Finite-Element-Methode. Akademie Verlag, 1993.

[14] N. Heuer, M.E. Mellado, and E.P. Stephan. $h p$-adaptive two-level methods for boundary integral equations on curves. Computing, 67(4):305-334, 2001.

[15] V. Heuveline and R. Rannacher. Duality-based adaptivity in the $h p$-finite element method. J. Numer. Math., 11(2), 2003.

[16] L. Hörmander. An Introduction Complex Analysis in Several Variables. North Holland, 1990.

[17] P. Houston, C. Schwab, and E. Süli. On the design of $h p$-adaptive finite element methods for elliptic partial differential equations. Comput. Meth. Appl. Mech. Engrg., (in press), 2004.

[18] G.E. Karniadakis and S.J. Sherwin. Spectral/hp Element Methods for CFD. Oxford University Press, 1999.

[19] Catherine Mavriplis. Adaptive mesh strategies for the spectral element method. Comput. Methods Appl. Mech. Engrg., 116(1-4):77-86, 1994. ICOSAHOM'92 (Montpellier, 1992).

[20] J.M. Melenk. hp finite element methods for singular perturbations, volume 1796 of Lecture Notes in Mathematics. Springer Verlag, 2002.

[21] J.M. Melenk, K. Gerdes, and C. Schwab. Fully discrete $h p$-FEM: fast quadrature. Comput. Meth. Appl. Mech. Engrg., 190:4339-4364, 2001.

[22] J.M. Melenk and B. Wohlmuth. On residual-based a posteriori error estimation in hp-FEM. Advances in Comp. Math., 15:311-331, 2001.

[23] J.T. Oden, W. Wu, and M. Ainsworth. Three-step $h p$ adaptive strategy for the incompressible Navier-Stokes equations. In I. Babuška, J. Flaherty, W. Henshaw, J. Hopcroft, J. Oliger, and T. Tezduyar, editors, Modeling, Mesh Generation, and Adaptive Numerical Methods for Partial Differential Equations, pages 347-366. Springer-Verlag, 1995. 
[24] T.J. Oden, A. Patra, and Y.S. Feng. An $h p$ adaptive strategy. In A.K. Noor, editor, Adaptive, Multilevel and Hierarchical Computational Strategies, pages 23-46. ASME Publications, 1993.

[25] C. Schwab. p- and hp-Finite Element Methods. Oxford University Press, 1998. 
Other titles in the SFB393 series:

03-01 E. Creusé, G. Kunert, S. Nicaise. A posteriory error estimation for the Stokes problem: Anisotropic and isotropic discretizations. January 2003.

03-02 S. I. Solov'ëv. Existence of the guided modes of an optical fiber. January 2003.

03-03 S. Beuchler. Wavelet preconditioners for the p-version of the FEM. February 2003.

03-04 S. Beuchler. Fast solvers for degenerated problems. February 2003.

03-05 A. Meyer. Stable calculation of the Jacobians for curved triangles. February 2003.

03-06 S. I. Solov'ëv. Eigenvibrations of a plate with elastically attached load. February 2003.

03-07 H. Harbrecht, R. Schneider. Wavelet based fast solution of boundary integral equations. February 2003.

03-08 S. I. Solov'ëv. Preconditioned iterative methods for monotone nonlinear eigenvalue problems. March 2003.

03-09 Th. Apel, N. Düvelmeyer. Transformation of hexahedral finite element meshes into tetrahedral meshes according to quality criteria. May 2003.

03-10 H. Harbrecht, R. Schneider. Biorthogonal wavelet bases for the boundary element method. April 2003.

03-11 T. Zhanlav. Some choices of moments of refinable function and applications. June 2003.

03-12 S. Beuchler. A Dirichlet-Dirichlet DD-pre-conditioner for p-FEM. June 2003.

03-13 Th. Apel, C. Pester. Clément-type interpolation on spherical domains - interpolation error estimates and application to a posteriori error estimation. July 2003.

03-14 S. Beuchler. Multi-level solver for degenerated problems with applications to p-version of the fem. (Dissertation) July 2003.

03-15 Th. Apel, S. Nicaise. The inf-sup condition for the Bernardi-Fortin-Raugel element on anisotropic meshes. September 2003.

03-16 G. Kunert, Z. Mghazli, S. Nicaise. A posteriori error estimation for a finite volume discretization on anisotropic meshes. September 2003.

03-17 B. Heinrich, K. Pönitz. Nitsche type mortaring for singularly perturbed reaction-diffusion problems. October 2003.

03-18 S. I. Solov'ëv. Vibrations of plates with masses. November 2003.

03-19 S. I. Solov'ëv. Preconditioned iterative methods for a class of nonlinear eigenvalue problems. November 2003.

03-20 M. Randrianarivony, G. Brunnett, R. Schneider. Tessellation and parametrization of trimmed surfaces. December 2003.

04-01 A. Meyer, F. Rabold, M. Scherzer. Efficient Finite Element Simulation of Crack Propagation. February 2004.

04-02 S. Grosman. The robustness of the hierarchical a posteriori error estimator for reactiondiffusion equation on anisotropic meshes. March 2004. 
04-03 A. Bucher, A. Meyer, U.-J. Görke, R. Kreißig. Entwicklung von adaptiven Algorithmen für nichtlineare FEM. April 2004.

04-04 A. Meyer, R. Unger. Projection methods for contact problems in elasticity. April 2004.

04-05 T. Eibner, J. M. Melenk. A local error analysis of the boundary concentrated FEM. May 2004.

04-06 H. Harbrecht, U. Kähler, R. Schneider. Wavelet Galerkin BEM on unstructured meshes. May 2004.

04-07 M. Randrianarivony, G. Brunnett. Necessary and sufficient conditions for the regularity of a planar Coons map. May 2004.

04-08 P. Benner, E. S. Quintana-Ortí, G. Quintana-Ortí. Solving Linear Matrix Equations via Rational Iterative Schemes. October 2004.

The complete list of current and former preprints is available via http://www.tu-chemnitz.de/sfb393/preprints.html. 